TRANSACTIONS OF THE

AMERICAN MATHEMATICAL SOCIETY

Volume 365, Number 12, December 2013, Pages 6669-6695

S 0002-9947(2013)06027-8

Article electronically published on August 15, 2013

\title{
PERELMAN'S ENTROPY AND KÄHLER-RICCI FLOW ON A FANO MANIFOLD
}

\author{
GANG TIAN, SHIJIN ZHANG, ZHENLEI ZHANG, AND XIAOHUA ZHU
}

\begin{abstract}
In this paper, we extend the method in a recent paper of Tian and Zhu to study the energy level $L(\cdot)$ of Perelman's entropy $\lambda(\cdot)$ for the Kähler-Ricci flow on a Fano manifold $M$. We prove that $L(\cdot)$ is independent of the initial metric of the Kähler-Ricci flow under an assumption that the modified Mabuchi's K-energy is bounded from below on $M$. As an application of the above result, we give an alternative proof to the main theorem about the convergence of Kähler-Ricci flow found in a 2007 paper by Tian and Zhu.
\end{abstract}

\section{INTRODUCTION}

In this paper, we extend the method in [TZhu5] to study the energy level $L(\cdot)$ of Perelman's entropy $\lambda(\cdot)$ for the Kähler-Ricci flow on an $n$-dimensional compact Kähler manifold $(M, J)$ with positive first Chern class $c_{1}(M)>0$ (namely $M$, called a Fano manifold). We will show that $L(\cdot)$ is independent of initial Kähler metrics in $2 \pi c_{1}(M)$ under an assumption that the modified Mabuchi's K-energy $\mu(\cdot)$ is bounded from below; see Proposition 3.1 in Section 3. The modified Mabuchi's K-energy $\mu(\cdot)$ is a generalization of Mabuchi's K-energy. It was showed in [TZhu2] that $\mu(\cdot)$ is bounded from below if $M$ admits a Kähler-Ricci soliton.

The proof of Proposition 3.1 depends on certain asymptotic estimates for minimizing functions (we call $f_{t}$-functions) defined by Perelman's $W$-functional associated to evolved metrics $g_{t}$ of the Kähler-Ricci flow (cf. Sections 2, 3, 4). We can compute the exact quantity $L(\cdot)=(2 \pi)^{-n}\left[n V-N_{X}\left(c_{1}(M)\right)\right]$, where $N_{X}\left(c_{1}(M)\right)$ is a nonnegative invariant depending only on the Kähler class $2 \pi c_{1}(M)$ and an extremal holomorphic vector field $X$ defined for Kähler-Ricci solitons on $(M, J)$. Moreover, $N_{X}\left(c_{1}(M)\right)$ is zero iff the Futaki-invariant vanishes (cf. Lemma 1.3). The quantity $(2 \pi)^{-n}\left[n V-N_{X}\left(c_{1}(M)\right)\right]$ is in fact the supremum of $\lambda(\cdot)$ in a class of $K_{X}$-invariant Kähler metrics in $2 \pi c_{1}(M)$ which can be achieved by a KählerRicci soliton on $(M, J)$ (cf. Corollary 1.5, Corollary 3.2). Here $K_{X}$ denotes a one-parameter compact subgroup of a holomorphism transformation group of $M$ generated by $X$.

As an application of Proposition 3.1, we prove the following convergence result about Kähler-Ricci flow.

Received by the editors January 30, 2012 and, in revised form, June 22, 2012 and August 29, 2012

2010 Mathematics Subject Classification. Primary 53C25; Secondary 53C55, 58J05.

Key words and phrases. Kähler-Ricci flow, Kähler-Ricci solitons, Perelman's entropy.

The third author was supported in part by a grant of BMCE 11224010007 in China.

The fourth author was supported in part by NSFC Grants 10990013 and 11271022. 
Theorem 0.1. Let $(M, J)$ be a compact Kähler manifold which admits a KählerRicci soliton $\left(g_{K S}, X\right)$. Then Kähler-Ricci flow with any initial Kähler metric in $\mathcal{K}_{X}$ will converge to a Kähler-Ricci soliton in $C^{\infty}$ in the sense of Kähler potentials. Moreover, the convergence can be made fast exponentially.

We note that without loss of generality we may assume that a Kähler-Ricci soliton $g_{K S}$ on $M$ is associated to the above $X$ (cf. [TZhu1, [TZhu2]). Theorem 0.1 was first proved by Tian and Zhu in TZhu3 by using an inequality of MoserTrudinger type established in [CTZ, 1 Here we will modify arguments in [TZhu5] in our general case that $(M, J)$ admits a Kähler-Ricci soliton to give an alternative proof of Theorem 0.1. This new proof does not use such an inequality of MoserTrudinger type. Moreover, in particular, in the case that $(M, J)$ admits a KählerEinstein metric, this new proof allows us to avoid the use of a deep result recently proved by Chen and Sun in [CS] for the uniqueness of Kähler-Einsteins in the sense of an orbit space to give a self-contained proof to the Main Theorem in [TZhu5].

The organization of the paper is as follows. In Section 1, we discuss an upper bound of $\lambda(\cdot)$ in the general case, without any condition for $\mu(\cdot)$, and we show that the quantity $(2 \pi)^{-n}\left[n V-N_{X}\left(c_{1}(M)\right)\right]$ is an upper bound of $\lambda(\cdot)$ in $\mathcal{K}_{X}$ (cf. Proposition 1.4). In Section 2, we will summarize to give some estimates for modified Ricci potentials of evolved Kähler metrics along the Kähler-Ricci flow (cf. Proposition 2.3). In Section 3, we prove Proposition 3.1. Theorem 0.1 will be proved in Section 6. In Section 4, we improve our key Lemma 3.3 in Section 3 independent of time $t$ (cf. Proposition 4.2). Section 5 is a discussion about an upper bound of $\lambda(\cdot)$ in $\mathcal{K}_{Y}$ for a general holomorphic vector field $Y$. Section 7 , Section 8 and Section 9 are three appendices about the gradient estimate and Laplace estimate for $f_{t}$, a proof of Lemma 6.1. and an a priori estimate about the local regularity of the Kähler-Ricci flow, respectively.

\section{AN UPPER BOUND OF $\lambda(\cdot)$}

In this section, we first review Perelman's $W$-functional for triples $(g, f, \tau)$ on a closed $m$-dimensional Riemannian manifold $M$ (cf. [Pe, [TZhu5]). Here $g$ is a Riemannian metric, $f$ is a smooth function and $\tau$ is a constant. In our situation, we will normalize the volume of $g$ by

$$
\int_{M} d V_{g} \equiv V
$$

and so we can fix $\tau$ by $\frac{1}{2}$. Then the $W$-functional depends only on a pair $(g, f)$ and it can be reexpressed as follows:

$$
W(g, f)=(2 \pi)^{-m / 2} \int_{M}\left[\frac{1}{2}\left(R(g)+|\nabla f|^{2}\right)+f\right] e^{-f} d V_{g},
$$

where $R(g)$ is a scalar curvature of $g$ and $(g, f)$ satisfies a normalization condition

$$
\int_{M} e^{-f} d V_{g}=V
$$

\footnotetext{
${ }^{1}$ We need to add more details about how to use the Moser-Trudinger type inequality in the general case.
} 
Then Perelman's entropy $\lambda(g)$ is defined by

$$
\lambda(g)=\inf _{f}\{W(g, f) \mid(g, f) \text { satisfies }(1.3)\} .
$$

It is well known that $\lambda(g)$ can be attained by some smooth function $f$ (cf. [Ro]). In fact, such an $f$ satisfies the Euler-Lagrange equation of $W(g, \cdot)$,

$$
\triangle f+f+\frac{1}{2}\left(R-|\nabla f|^{2}\right)=(2 \pi)^{m / 2} V^{-1} \lambda(g) .
$$

Following Perelman's computation in [Pe], we can deduce the first variation of $\lambda(g)$,

$$
\delta \lambda(g)=-(2 \pi)^{-m / 2} \int_{M}\left\langle\delta g, \operatorname{Ric}(g)-g+\nabla^{2} f\right\rangle e^{-f} d V_{g}
$$

where $\operatorname{Ric}(g)$ denotes the Ricci tensor of $g$ and $\nabla^{2} f$ is the Hessian of $f$. Hence, $g$ is a critical point of $\lambda(\cdot)$ if and only if $g$ is a gradient shrinking Ricci soliton which satisfies

$$
\operatorname{Ric}(g)+\nabla^{2} f=g,
$$

where $f$ is a minimizer of $W(g, \cdot)$. The following lemma was proved in [TZhu5] for the uniqueness of solutions (1.4) when $g$ is a gradient shrinking Ricci soliton.

Lemma 1.1. If $g$ satisfies (1.6) for some $f$, then any solution of (1.4) is equal to $f$ modulo a constant. Consequently, a minimizer of $W(\mathrm{~g}, \cdot)$ is unique if the metric $g$ is a gradient shrinking Ricci soliton. Conversely, if $f$ is a function in (1.6) for $g$, then $f$ satisfies (1.4).

In the case that $(M, J)$ is an $n$-dimensional Fano manifold, for any Kähler metric $g$ in $2 \pi c_{1}(M)$, (1.1) is equal to

$$
\int_{M} d V_{g}=\int_{M} \omega_{g}^{n}=(2 \pi)^{n} \int_{M} c_{1}(M)^{n} \equiv V .
$$

Moreover, (1.6) becomes an equation of Kähler-Ricci solitons,

$$
\operatorname{Ric}\left(\omega_{g}\right)-\omega_{g}=\mathrm{L}_{X} \omega_{g}
$$

where $\operatorname{Ric}\left(\omega_{g}\right)$ is a Ricci form of $g$ and $L_{X}$ denotes the Lie derivative along a holomorphic vector field $X$ on $M$. By the uniqueness of Kähler-Ricci solitons [TZhu1], TZhu2], we may assume that $X$ lies in a reductive Lie subalgebra $\eta_{r}(M)$ of $\eta(M)$ after a holomorphism transformation, where $\eta(M)$ consists of all holomorphic vector fields on $M$. Such an $X$ (we call it an extremal holomorphic vector field for Kähler-Ricci solitons) can be determined as follows.

Let $\operatorname{Aut}_{r}(M)$ be a connected Lie subgroup of an automorphism group of $M$ generated by $\eta_{r}(M)$. Let $K$ be a maximal compact subgroup of $\operatorname{Aut}_{r}(M)$. Without loss of generality, we may choose a $K$-invariant background metric $g$ with its Kähler form $\omega_{g}$ in $2 \pi c_{1}(M)$. In [TZhu2, as an obstruction to Kähler-Ricci solitons, Tian and Zhu introduced a modified Futaki-invariant $F_{X}(v)$ for any $X, v \in \eta(M)$ by

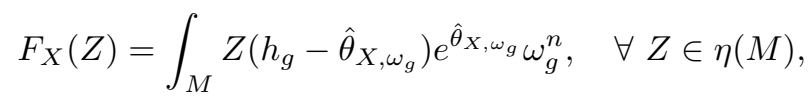

where $h_{g}$ is a Ricci potential of $g$ and $\hat{\theta}_{X, \omega_{g}}$ is a complex-valued potential of $X$ associated to $g$ defined by $L_{X} \omega_{g}=\sqrt{-1} \partial \bar{\partial} \hat{\theta}_{X, \omega_{g}}$ with a normalization condition

$$
\int_{M} \hat{\theta}_{X, \omega_{g}} e^{h_{g}} \omega_{g}^{n}=0
$$


It was shown that there exists a unique $X \in \eta_{r}(M)$ with its real-valued potential $\hat{\theta}_{X, \omega_{g}}$ such that

$$
F_{X}(v) \equiv 0, \forall v \in \eta_{r}(M) .
$$

Moreover, $F_{X}(v) \equiv 0$ for any $v \in \eta(M)$ if $(M, J)$ admits a Kähler-Ricci soliton.

Let $K_{X}$ be a one-parameter compact subgroup of a holomorphism transformation group generated by $X$. We denote $\mathcal{K}_{X}$ to be a class of $K_{X}$-invariant Kähler metrics in $2 \pi c_{1}(M)$. Let $\theta_{X, \omega_{g}}$ be a real-valued potential of $X$ associated to $g$ with a normalization condition

$$
\int_{M} e^{\theta X, \omega_{g}} \omega_{g}^{n}=\int_{M} \omega_{g}^{n}=V
$$

Clearly, $\theta_{X, \omega_{g}}=\hat{\theta}_{X, \omega_{g}}-c_{X}$ for some constant $c_{X}$ which is independent of $g \in \mathcal{K}_{X}$.

Definition 1.2. For $g \in \mathcal{K}_{X}$, define $N_{X}\left(\omega_{g}\right)$ by

$$
N_{X}\left(\omega_{g}\right)=\int_{M} \theta_{X, \omega_{g}} e^{\theta_{X}, \omega_{g}} \omega_{g}^{n} .
$$

By Jensen's inequality, it is easy to see

$$
\begin{aligned}
& \frac{1}{V} \int_{M}\left(-\theta_{X, \omega_{g}}\right) e^{\theta_{X, \omega_{g}} \omega_{g}^{n}} \\
& \leq \log \left\{\frac{1}{V} \int_{M} e^{-\theta_{X, \omega_{g}}} e^{\left.\theta_{X, \omega_{g}} \omega_{g}^{n}\right\}}=0 .\right.
\end{aligned}
$$

The equality holds if and only if $\theta_{X, \omega_{g}}=0$. This shows that $N_{X}\left(\omega_{g}\right)$ is nonnegative and it is zero if and only if the Futaki-invariant vanishes $\mathrm{Fu}$. Moreover, we have

Lemma 1.3. $N_{X}\left(\omega_{g}\right)$ is independent of the choice of $g$ in $\mathcal{K}_{X}$.

Proof. Choose a $K$-invariant Kähler form $\omega$ in $2 \pi c_{1}(M)$. Then for any Kähler metric $g$ in $\mathcal{K}_{X}$ there exists a Kähler potential $\varphi$ such that the imaginary part of $X(\varphi)$ vanishes and the Kähler form of $g$ satisfies

$$
\omega_{g}=\omega_{\varphi}=\omega+\sqrt{-1} \partial \bar{\partial} \varphi
$$

Thus we suffice to prove

$$
N_{X}\left(\omega_{\varphi}\right)=N_{X}\left(\omega_{t \varphi}\right), \forall t \in[0,1],
$$

where $\omega_{t \varphi}=\omega+t \sqrt{-1} \partial \bar{\partial} \varphi$. This follows from

$$
\begin{aligned}
\frac{d N_{X}\left(\omega_{t \varphi}\right)}{d t} & =\int_{M} X(\varphi) e^{\theta_{X, \omega_{t}}} \omega_{t \varphi}^{n}+\int_{M} \theta_{X, \omega_{t \varphi}}(X+\triangle)(\varphi) e^{\theta X, \omega_{t \varphi}} \omega_{t \varphi}^{n} \\
& =\int_{M} X(\varphi) e^{\theta_{X, \omega_{t}}} \omega_{t \varphi}^{n}-\int_{M} \nabla^{i} \varphi \nabla_{\bar{i}} \theta_{X, \omega_{t \varphi}} \omega_{t \varphi}^{n} \\
& =0 .
\end{aligned}
$$

Here we have used the fact

$$
\theta_{X, \omega_{t \varphi}}=\theta_{X, \omega_{g}}+t X(\varphi) .
$$

By the above lemma, $N_{X}(\cdot)$ is an invariant on $\mathcal{K}_{X}$, which is independent of the choice of $g$. For simplicity, we denote this invariant by $N_{X}\left(c_{1}(M)\right)$. The following proposition gives an upper bound of $\lambda(\cdot)$ in $\mathcal{K}_{X}$ related to $N_{X}\left(c_{1}(M)\right)$. 


\section{Proposition 1.4.}

$$
\sup _{g \in \mathcal{K}_{X}} \lambda(g) \leq(2 \pi)^{-n}\left[n V-N_{X}\left(c_{1}(M)\right)\right] .
$$

Proof. Since $\lambda(g) \leq W\left(g,-\theta_{X, \omega_{g}}\right)$, we suffice to prove

$$
W\left(g,-\theta_{X, \omega_{g}}\right)=(2 \pi)^{-n}\left[n V-N_{X}\left(c_{1}(M)\right)\right] .
$$

In fact, by using the facts $R(g)=2 n+\Delta h_{g}$ and

$$
\int_{M}\left(\Delta \theta_{X, \omega_{g}}+\left|\nabla \theta_{X, \omega_{g}}\right|^{2}\right) e^{\theta_{X, \omega_{g}} \omega_{g}^{n}=0}
$$

we have

$$
\begin{aligned}
& \int_{M}\left(R(g)+\left|\nabla \theta_{X, \omega_{g}}\right|^{2}\right) e^{\theta_{X, \omega_{g}} \omega_{g}^{n}} \\
& =2 n V+\int_{M}\left(\Delta h_{g}-\Delta \theta_{X, \omega_{g}}\right) e^{\theta_{X, \omega_{g}} \omega_{g}^{n}} \\
& =2 n V-\int_{M}\left\langle\nabla\left(h_{g}-\theta_{X, \omega_{g}}\right), \nabla \theta_{X, \omega_{g}}\right\rangle e^{\theta_{X, \omega_{g}} \omega_{g}^{n}} \\
& =2 n V-2 \int_{M} X\left(h_{g}-\theta_{X, \omega_{g}}\right) e^{\theta_{X, \omega_{g}} \omega_{g}^{n}} \\
& =2 n V-2 e^{-c_{X}} F_{X}(X) .
\end{aligned}
$$

In the last equality above, we used the relation (1.8). Since $X$ is extremal, we have

$$
F_{X}(X)=0 .
$$

Thus by (1.2) for $f=-\theta_{X, \omega}$, together with Lemma 1.3, one will get (1.11).

In the case that $M$ admits a Kähler-Ricci soliton $g_{K S}$, by Lemma 1.1, a minimizer $f$ of $W\left(g_{K S}, \cdot\right)$ in $\mathcal{K}_{X}$ must be $-\theta_{X}$. Thus for any $g \in \mathcal{K}_{X}$, by Proposition 1.4 , we have

$$
\begin{aligned}
\lambda\left(g_{K S}\right) & =W\left(g_{K S},-\theta_{X}\right) \\
& =(2 \pi)^{-n}\left[n V-N_{X}\left(c_{1}(M)\right)\right] \geq \lambda(g) .
\end{aligned}
$$

Therefore we get the following corollary.

Corollary 1.5. Suppose that $(M, J)$ admits a Kähler-Ricci soliton $g_{K S}$. Then $g_{K S}$ is a global maximizer of $\lambda(\cdot)$ in $\mathcal{K}_{X}$ and

$$
\lambda\left(g_{K S}\right)=(2 \pi)^{-n}\left[n V-N_{X}\left(c_{1}(M)\right)\right] .
$$

Remark 1.6. Corollary 1.5 implies that a Kähler-Einstein metric is a global maximizer of $\lambda(\cdot)$ in $2 \pi c_{1}(M)$, even with varying complex structures and a supremum of $\lambda(\cdot)(2 \pi)^{-n} n V$ since $N_{X}\left(c_{1}(M)\right)=0$. Note that $N_{X}\left(c_{1}(M)\right)>0$ if the Futakiinvariant does not vanish. Thus Corollary [1.5] also implies that the supremum of $\lambda(\cdot)$ in the case that $(M, J)$ admits a Kähler-Ricci soliton is strictly less than one in the case that $(M, J)$ admits a Kähler-Einstein metric. 


\section{Estimates for modified Ricci potentials}

In this section, we summarize some a priori estimates for modified Ricci potentials of evolved Kähler metrics along the Kähler-Ricci flow. Some similar estimates have also been discussed in [TZhu3] and PSSW]; we refer the readers to those two papers. We consider the following (normalized) Kähler-Ricci flow:

$$
\frac{\partial g(t, \cdot)}{\partial t}=-\operatorname{Ric}(g(t, \cdot))+g(t, \cdot), g(0)=g
$$

where $g$ is an initial Kähler metric with its Kähler form in $2 \pi c_{1}(M)$. It was proved in [Ca] that (2.1) has a global solution $g_{t}=g(t, \cdot)$ for all time $t>0$. For simplicity, we denote by $\left(g_{t} ; g\right)$ a solution of (2.1) with the initial metric $g$. Since the flow preserves the Kähler class, we may write the Kähler form of $g_{t}$ as

$$
\omega_{\phi}=\omega_{g}+\sqrt{-1} \partial \bar{\partial} \phi
$$

for some Käher potential $\phi=\phi_{t}$.

Let $X \in \eta_{r}(M)$ be the extremal holomorphic vector field on $M$ as in Section 1 and $\sigma_{t}=\exp \{t X\}$ be a one-parameter subgroup generated by $X$. Let $\phi^{\prime}=\phi_{\sigma_{t}}$ be the corresponding Kähler potentials of $\sigma_{t}^{\star} \omega_{\phi_{t}}$. Then $\omega_{\phi^{\prime}}$ will satisfy a modified Kähler-Ricci flow,

$$
\frac{\partial}{\partial t} \omega_{\phi^{\prime}}=-\operatorname{Ric}\left(\omega_{\phi^{\prime}}\right)+\omega_{\phi^{\prime}}+L_{X} \omega_{\phi^{\prime}}
$$

Equation (2.2) is equivalent to the following Monge-Ampère flow for $\phi^{\prime}$ (modulo a constant):

$$
\frac{\partial \phi^{\prime}}{\partial t}=\log \frac{\omega_{\phi^{\prime}}^{n}}{\omega_{g}^{n}}+\phi^{\prime}+\theta_{X, \omega_{\phi^{\prime}}}-h_{g}, \phi^{\prime}(0, \cdot)=c,
$$

where $c$ is a constant and all Kähler potentials $\phi^{\prime}=\phi_{t}^{\prime}=\phi^{\prime}(t, \cdot)$ are in a space given by

$$
\mathcal{P}_{X}(M, \omega)=\left\{\varphi \in C^{\infty}(M) \mid \omega_{\varphi}=\omega+\sqrt{-1} \partial \bar{\partial} \varphi>0, \quad \operatorname{Im}(X(\varphi))=0\right\} .
$$

By using the maximum principle to (2.2) or (2.3), we get

$$
h_{\phi^{\prime}}-\theta_{X, \omega_{\phi^{\prime}}}=-\frac{\partial}{\partial t} \phi^{\prime}+c_{t}
$$

for some constants $c_{t}$. Here $h_{\phi^{\prime}}$ are Ricci potentials of $\omega_{\phi^{\prime}}$ which are normalized by

$$
\int_{M} e^{h_{\phi^{\prime}}} \omega_{\phi^{\prime}}^{n}=V
$$

The following estimates are due to G. Perelman. We refer the readers to $[\underline{S T}$ for their proof.

Lemma 2.1. There are constants $c>0$ and $C>0$ depending only on the initial metric $g$ such that (a) $\operatorname{diam}\left(M, \omega_{\phi^{\prime}}\right) \leq C$; (b) $\operatorname{vol}\left(B_{r}(p), \omega_{\phi^{\prime}}\right) \geq c r^{2 n}$; (c) $\left\|h_{\phi^{\prime}}\right\|_{C^{0}(M)} \leq C ;(d)\left\|\nabla h_{\phi^{\prime}}\right\|_{\omega_{\phi^{\prime}}} \leq C ;(e)\left\|\Delta h_{\phi^{\prime}}\right\|_{C^{0}(M)} \leq C$.

Recall that the modified Mabuchi's K-energy $\mu(\cdot)$ is defined in $\mathcal{P}_{X}(M, \omega)$ by

$$
\begin{aligned}
\mu(\varphi)= & -\frac{n}{V} \int_{0}^{1} \int_{M} \dot{\psi}\left[\operatorname{Ric}\left(\omega_{\psi}\right)-\omega_{\psi}-\sqrt{-1} \partial \bar{\partial} \theta_{X, \omega_{\psi}}\right. \\
& \left.+\sqrt{-\partial}\left(h_{\omega_{\psi}}-\theta_{X, \omega_{\psi}}\right) \wedge \partial \theta_{X, \omega_{\psi}}\right] \wedge e^{\theta_{X, \omega_{\psi}} \omega_{\psi}^{n-1} \wedge d t},
\end{aligned}
$$


where $\psi=\psi_{t}(0 \leq t \leq 1)$ is a path connecting 0 to $\varphi$ in $\mathcal{P}_{X}(M, \omega)$. If $X=0$, then $\mu_{\omega_{g}}(\phi)$ is nothing but Mabuchi's K-energy Ma. Then by (2.2), we have

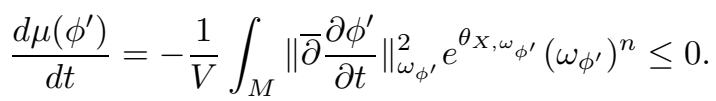

This implies that $\mu\left(\phi^{\prime}\right)$ is uniformly bounded if $\mu(\cdot)$ is bounded from below in $\mathcal{P}_{X}(M, \omega)$.

Let $u_{X, \phi^{\prime}}=u_{X, \omega_{g_{t}^{\prime}}}=h_{\phi^{\prime}}-\theta_{X, \omega_{\phi^{\prime}}}$. Then

Lemma 2.2. There exists a uniform $C$ such that

$$
\left\|\nabla u_{X, \phi^{\prime}}\right\|_{\omega_{\phi^{\prime}}} \leq C \text {. }
$$

Proof. First we note that $\theta_{X, \omega_{\phi^{\prime}}}$ is uniformly bounded in $\mathcal{P}_{X}(M, \omega)$ [Zhu1], [ZZ]. Then by (c) of Lemma 2.1, we have

$$
\left\|u_{X, \phi^{\prime}}\right\|_{C^{0}}=\left\|u_{X, \omega_{g_{s}^{\prime}}}\right\|_{C^{0}} \leq C, \forall s>0
$$

for some uniform constant $C$. Now we consider the flow 2.3) with zero as an initial Kähler potential and the background Kähler form $\omega_{g}$ replaced by $\omega_{g_{s}^{\prime}}$. By an estimate in Lemma 4.3 in CTZ, we see

$$
t\left\|\nabla u_{X, \omega_{g_{s+t}^{\prime}}}\right\|_{\omega_{g_{s+t}^{\prime}}}^{2} \leq e^{2 t}\left\|u_{X, \omega_{g_{s}^{\prime}}}\right\|_{C^{0}}, \forall t>0 .
$$

In particular, we get

$$
\left\|\nabla u_{X, \omega_{g_{s+t}^{\prime}}}\right\|_{\omega_{g_{s+t}^{\prime}}}^{2} \leq C^{\prime}, \forall t \in[1,2] .
$$

Since the above estimate is independent of $s$, we conclude that the lemma is true.

Now we begin to prove the main result in this section.

Proposition 2.3. Suppose that $\mu(\cdot)$ is bounded from below in $\mathcal{P}_{X}(M, \omega)$. Then we have:

(a) $\lim _{t \rightarrow \infty}\left\|u_{X, \phi^{\prime}}\right\|_{C^{0}}=0$;

(b) $\lim _{t \rightarrow \infty}\left\|\nabla u_{X, \phi^{\prime}}\right\|_{\omega_{\phi^{\prime}}}=0$;

(c) $\lim _{t \rightarrow \infty}\left\|\triangle u_{X, \phi^{\prime}}\right\|_{C^{0}}=0$.

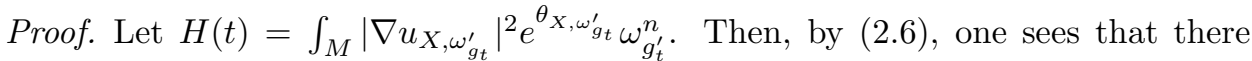
exists a sequence of $t_{i} \in[i, i+1]$ such that

$$
\lim _{i \rightarrow \infty} H\left(t_{i}\right)=0 .
$$

Thus by using a differential inequality

$$
\frac{d H(t)}{d t} \leq C H(t)
$$

where $C$ is a uniform constant (cf. [PSSW]), we get

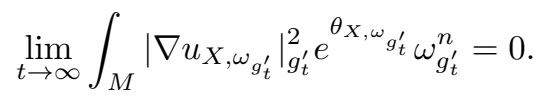

Let

$$
\tilde{u}_{t}=u_{X, \omega_{g_{t}^{\prime}}}-\frac{1}{V} \int_{M} u_{X, \omega_{g_{t}^{\prime}}} e^{h_{t}^{\prime}} \omega_{g_{t}^{\prime}}^{n}
$$


where $h_{t}^{\prime}=h_{\phi^{\prime}(t, \cdot)}$. Then by using the weighted Poincaré inequality in [TZhu3] together with (c) of Lemma 2.1, we obtain from (2.7),

$$
\int_{M} \tilde{u}_{t}^{2} e^{h_{t}^{\prime}} \omega_{g_{t}^{\prime}}^{n} \leq \int_{M}\left|\nabla u_{X, \omega_{g_{t}^{\prime}}}\right|_{g_{t}^{\prime}}^{2} e^{h_{t}^{\prime}} \omega_{g_{t}^{\prime}}^{n} \rightarrow 0, \text { as } t \rightarrow \infty
$$

Consequently, we derive

$$
\lim _{t \rightarrow \infty} \int_{M} \tilde{u}_{t}^{2} \omega_{g_{t}^{\prime}}^{n}=0
$$

We claim

$$
\lim _{t \rightarrow \infty}\left\|\tilde{u}_{t}\right\|_{C^{0}}=0 .
$$

The claim immediately implies (a) of Proposition 2.3 by the normalization conditions

$$
\int_{M} e^{\theta x, \omega} g_{t}^{\prime} \omega_{g_{t}^{\prime}}^{n}=\int_{M} e^{h_{t}^{\prime}} \omega_{g_{t}^{\prime}}^{n}=V
$$

To prove the claim, we need to use an inequality

$$
\left\|\tilde{u}_{t}\right\|_{C^{0}}^{n+1} \leq C\left\|\nabla u_{X, \omega_{g_{t}^{\prime}}}\right\|_{g_{t}^{\prime}}^{n}\left[\int_{M} \tilde{u}_{t}^{2} \omega_{g_{t}^{\prime}}^{n}\right]^{\frac{1}{2}} .
$$

(2.9) can be proved by using the noncollapsing estimate (b) in Lemma 2.1 (cf. [PSSW], Zhu2]). Thus by Lemma 2.2 and (2.8), the claim is proved.

By (a) we can show that after a suitable choice of constant $c$ in the flow (2.3) it holds that

$$
\lim _{t \rightarrow \infty}\left\|\frac{\partial}{\partial t} \phi^{\prime}\right\|_{C^{0}}=0
$$

In fact, under the assumption of a lower bound of modified $K$-energy, one can choose such a $c$ (cf. TZhu3 ) such that

$$
\lim _{t \rightarrow \infty} \int_{M} \frac{\partial}{\partial t} \phi^{\prime} e^{\theta_{X, \omega} \phi_{\phi^{\prime}}} \omega_{\phi^{\prime}}^{n}=0 .
$$

Then, by (2.4), we will get the conclusion. On the other hand, by Lemma 2.2 and (d) of Lemma 2.1, we have

$$
\sup _{t \in[0, \infty)}\|X\|_{g_{t}^{\prime}}<C
$$

for some uniform constant $C$. Therefore, by using the following lemma we prove (b) and (c).

Lemma 2.4 ([יPSW] $)$. There exist $\delta, K>0$ depending only on $n$ and the constant $C_{X}=\sup _{t \in[0, \infty)}\|X\|_{g_{t}^{\prime}}$ with the following property. For $\epsilon$ with $0<\epsilon \leq \delta$ and any $t_{0}>0$, if

$$
\left\|\frac{\partial \phi^{\prime}}{\partial t}\right\|_{C^{0}}\left(t_{0}\right) \leq \epsilon
$$

then

$$
\left\|\nabla u_{X, \omega_{g_{t_{0}+2}^{\prime}}^{\prime}}\right\|_{g_{t_{0}+2}^{\prime}}^{2}+\left\|\Delta u_{X, \omega_{g_{t_{0}+2}^{\prime}}^{\prime}}\right\|_{C^{0}} \leq K \epsilon
$$




\section{Estimate for $L(\cdot)$}

According to [TZhu5, the energy level $L(g)$ of entropy $\lambda(\cdot)$ for the Kähler-Ricci flow $\left(g_{t} ; g\right)$ is defined by

$$
L(g)=\lim _{t \rightarrow \infty} \lambda\left(g_{t}\right)
$$

By the monotonicity of $\lambda\left(g_{t}\right)$, we see that $L(g)$ exists and is finite. In this section, our goal is to prove

Proposition 3.1. Suppose that the modified Mabuchi's K-energy is bounded from below in $\mathcal{K}_{X}$. Then for any $g \in \mathcal{K}_{X}$,

$$
L(g)=(2 \pi)^{-n}\left(n V-N_{X}\left(c_{1}(M)\right) .\right.
$$

Proposition 3.1 shows that $L(g)$ does not depend on the initial Kähler metric $g \in \mathcal{K}_{X}$. Thus by using the Kähler-Ricci flow $\left(g_{t} ; g\right)$ for any Kähler metric $g \in \mathcal{K}_{X}$ and the monotonicity of $\lambda\left(g_{t}\right)$, we get the following generalization of Corollary 1.5 .

Corollary 3.2. Suppose that the modified Mabuchi's K-energy is bounded from below. Then

$$
\sup \left\{\lambda\left(g^{\prime}\right) \mid g^{\prime} \in \mathcal{K}_{X}\right\}=(2 \pi)^{-n}\left[n V-N_{X}\left(c_{1}(M)\right)\right] .
$$

To prove Proposition 3.1, we need the following key lemma.

Lemma 3.3. Let $f_{t}$ be a minimizer of the $W\left(g_{t}, \cdot\right)$-functional associated evolved Kähler metric $g_{t}$ of (2.1) at time $t$ and $h_{t}$ a Ricci potential of $g_{t}$ which satisfies the normalization (2.5). Then there exists a sequence of $t_{i} \in[i, i+1]$ such that

(a) $\lim _{t_{i} \rightarrow \infty}\left\|\Delta\left(f_{t_{i}}+h_{t_{i}}\right)\right\|_{L^{2}\left(M, \omega_{g_{i}}\right)}=0$;

(b) $\lim _{t_{i} \rightarrow \infty}\left\|\nabla\left(f_{t_{i}}+h_{t_{i}}\right)\right\|_{L^{2}\left(M, \omega_{g_{i}}\right)}=0$;

(c) $\lim _{t_{i} \rightarrow \infty}\left\|f_{t_{i}}+h_{t_{i}}\right\|_{C^{0}}=0$.

Proof. Lemma 3.3 is a generalization of Proposition 4.4 in TZhu5. We will follow the argument there. First, by (1.5), it is easy to see that

$$
\frac{d}{d t} \lambda\left(g_{t}\right)=(2 \pi)^{-n} \int_{M}\left|\operatorname{Ric}\left(g_{t}\right)-g_{t}+\nabla^{2} f_{t}\right|_{g_{t}}^{2} e^{-f_{t}} \omega_{g_{t}}^{n} .
$$

It follows that

$$
\frac{d}{d t} \lambda\left(g_{t}\right) \geq(2 \pi)^{-n} \frac{1}{2 n} \int_{M}\left|\triangle\left(h_{t}+f_{t}\right)\right|^{2} e^{-f(t)} \omega_{g_{t}}^{n}
$$

Since $\lambda\left(g_{t}\right) \leq W\left(g_{t}, 0\right)=(2 \pi)^{-n} n V$ are uniformly bounded, we see that there exists a sequence of $t_{i} \in[i, i+1]$ such that

$$
\lim _{i \rightarrow \infty} \int_{M}\left|\triangle\left(h_{t_{i}}+f_{t_{i}}\right)\right|^{2} e^{-f_{t_{i}}} \omega_{g_{t_{i}}}^{n}=0 .
$$

Note that $f_{t}$ is uniformly bounded [TZhu5]. Hence we see that that (a) of the lemma is true. By (a), we also get

$$
\begin{aligned}
& \left.\lim _{t_{i} \rightarrow \infty}\left\|\nabla\left(f_{t_{i}}+h_{t_{i}}\right)\right\|_{L^{2}\left(M, \omega_{g_{i}}\right.}\right) \\
& \leq \lim _{t_{i} \rightarrow \infty} \int_{M}\left|f_{t_{i}}+h_{t_{i}} \| \triangle\left(f_{t_{i}}+h_{t_{i}}\right)\right| \omega_{g_{t_{i}}}^{n} \\
& \left.\leq C \lim _{t_{i} \rightarrow \infty}\left\|\Delta\left(f_{t_{i}}+h_{t_{i}}\right)\right\|_{L^{2}\left(M, \omega_{g_{t_{i}}}\right.}\right)=0 .
\end{aligned}
$$

This proves (b) of the lemma. It remains to prove (c). 
Let $q_{t}=f_{t}+h_{t}$. Then

$$
\begin{aligned}
-\Delta q_{t} & =-\Delta f_{t}-\Delta h_{t} \\
& =f_{t}+\frac{1}{2}\left(R-\left|\nabla f_{t}\right|^{2}\right)-(2 \pi)^{2 n} V^{-1} \lambda\left(g_{t}\right)-\Delta h_{t} \leq C .
\end{aligned}
$$

Define

$$
\tilde{q}_{t}=q_{t}-c(t)
$$

where $c(t)=\frac{1}{V} \int_{M} q_{t} e^{h_{t}} \omega_{g_{t}}^{n}$. By using the weighted Poincaré inequality [TZhu3], we have

It follows by (b) that

$$
\int_{M} \tilde{q}_{t}^{2} e^{h_{t}} \omega_{g_{t}}^{n} \leq \int_{M}\left|\nabla q_{t}\right|^{2} e^{h_{t}} \omega_{g_{t}}^{n}
$$

$$
\lim _{i \rightarrow \infty} \int_{M} \tilde{q}_{t_{i}}^{2} \omega_{g_{t_{i}}}^{n}=0 .
$$

Hence, following an argument in the proof of Proposition 4.4 in [TZhu5, we will get estimates

$$
\left\|\tilde{q_{t_{i}}}{ }^{+}\right\|_{C^{0}} \leq C\left\|\tilde{q}_{t_{i}}\right\|_{L^{2}\left(M, \omega_{g_{t_{i}}}\right)} \rightarrow 0, \text { as } i \rightarrow \infty
$$

and

$$
\lim _{i \rightarrow \infty} \int_{M} \tilde{q_{i}}-\omega_{g_{t_{i}}}^{n}=0,
$$

where $q_{t}^{+}=\max \left\{q_{t}, 0\right\}$ and $q_{t}^{-}=\min \left\{q_{t}, 0\right\}$. Consequently, we derive

$$
\lim _{i \rightarrow \infty} \int_{M} \tilde{q_{i}} e^{-f_{t_{i}}} \omega_{g_{t_{i}}}^{n}=0 .
$$

On the other hand, by (3.6) and (3.7), one sees that for any $\epsilon>0$

$$
\lim _{i \rightarrow \infty} \operatorname{mes} E_{i}(\epsilon)=0 \text {, }
$$

where $E_{i}(\epsilon)=\left\{x \in M|| \tilde{t_{i}} \mid \geq \epsilon\right\}$ and mes $E_{i}(\epsilon)=\int_{E_{i}(\epsilon)} \omega_{g_{t_{i}}}^{n}$. Then by the normalization $\int_{M} e^{-f_{t}} \omega_{g_{t}}^{n}=\int_{M} e^{h_{t}} \omega_{g_{t}}^{n}=V$ and the fact that $f_{t}$ and $g_{t}$ are uniformly bounded, it is easy to prove that

$$
\lim _{i \rightarrow \infty} c\left(t_{i}\right)=0 .
$$

This implies

$$
\lim _{i \rightarrow \infty} \int_{M} q_{t_{i}} e^{-f_{t_{i}}} \omega_{g_{t_{i}}}^{n}=0 .
$$

Next we improve (3.8) to

$$
\lim _{i \rightarrow \infty}\left\|q_{t_{i}}\right\|_{C^{0}}=0
$$

Let $u_{t}=e^{-\frac{f_{t}}{2}}-e^{\frac{h_{t}}{2}}$. We claim

$$
\lim _{i \rightarrow \infty}\left\|u_{t_{i}}\right\|_{L^{2}\left(M, \omega_{g_{t_{i}}}\right)}=0 .
$$

In fact, by Jensen's inequality and (3.8), one sees

$$
\begin{aligned}
\frac{1}{V} \int_{M} e^{-\frac{f_{t_{i}}}{2}} e^{\frac{h_{t_{i}}}{2}} \omega_{g_{t_{i}}}^{n} & =\frac{1}{V} \int_{M} e^{\frac{f_{t_{i}}+h_{t_{i}}}{2}} e^{-f_{t_{i}}} \omega_{g_{t_{i}}}^{n} \\
& \geq e^{\frac{1}{2 V} \int_{M}\left(f_{t_{i}}+h_{t_{i}}\right)} e^{-f_{t_{i}}} \omega_{g_{t_{i}}}^{n} \rightarrow 1, \text { as } i \rightarrow \infty .
\end{aligned}
$$


On the other hand,

Hence

$$
\int_{M} e^{-\frac{f_{t}}{2}} e^{\frac{h_{t}}{2}} \omega_{g_{t}}^{n} \leq\left(\int_{M} e^{-f_{t}} \omega_{g_{t}}^{n}\right)^{\frac{1}{2}}\left(\int_{M} e^{h_{t}} \omega_{g_{t}}^{n}\right)^{\frac{1}{2}}=V .
$$

It follows that

$$
\lim _{i \rightarrow \infty} \int_{M} e^{-\frac{f_{t_{i}}}{2}} e^{\frac{h_{t_{i}}}{2}} \omega_{g_{t_{i}}}^{n}=V
$$

$$
\lim _{i \rightarrow \infty} \int_{M} u_{t_{i}}^{2} \omega_{g_{t_{i}}}^{n}=2 V-2 \lim _{i \rightarrow \infty} \int_{M} e^{-\frac{f_{t_{i}}}{2}} e^{\frac{h_{t_{i}}}{2}} \omega_{g_{t_{i}}}^{n}=0 .
$$

This completes the proof of the claim.

Since equation (1.4) is equivalent to

$$
\Delta v_{t}-\frac{1}{2} f_{t} v_{t}-\frac{1}{4} R\left(g_{t}\right) v_{t}=\frac{1}{2 V}(2 \pi)^{n} \lambda\left(g_{t}\right) v_{t},
$$

where $v_{t}=e^{\frac{-f_{t}}{2}}$, by Lemma 2.1, it is easy to see that

$$
\left|\Delta u_{t}\right| \leq C \text {. }
$$

Then by the standard Moser's iteration, we get from (3.10) that

$$
\left\|u_{t_{i}}\right\|_{C^{0}} \leq C\left\|u_{t_{i}}\right\|_{L^{2}\left(M, \omega_{g_{t_{i}}}\right)} \rightarrow 0 \text {, as } i \rightarrow \infty .
$$

This implies (3.9), so we prove (c) of the lemma.

Proof of Proposition 3.1. Note that $\frac{R\left(g_{t}\right)}{2}=n+\frac{1}{2} \Delta h_{t}$, where $\Delta$ is the BeltrimaLaplacian operator associated to the Riemannian metric $g_{t}$. Then

$$
\int_{M} \frac{1}{2}\left(R\left(g_{t}\right)+\left|\nabla f_{t}\right|^{2}\right) e^{-f_{t}} d V_{g_{t}}=n V+\frac{1}{2} \int_{M} \Delta\left(f_{t}+h_{t}\right) e^{-f_{t}} d V_{g_{t}} .
$$

Thus by (a) of Lemma 3.3, one sees that there exists a sequence of time $t_{i}$ such that

$$
\lim _{i \rightarrow \infty} \int_{M} \frac{1}{2}\left(R\left(g_{t_{i}}\right)+\left|\nabla f_{t_{i}}\right|^{2}\right) e^{-f_{t_{i}}} d V_{g_{t_{i}}}=n V .
$$

On the other hand, since the modified Mabuchi's K-energy is bounded from below, we see that (a) of Proposition 2.3 is true. Then by (c) of Lemma 3.3, it follows that

$$
\lim _{i \rightarrow \infty}\left\|f_{t_{i}}+\theta_{X, \omega_{g_{t_{i}}}}\right\|_{C^{0}}=0 \text {. }
$$

Here we used the fact $\sigma_{t}^{\star} \theta_{X, \omega_{g_{t}}}=\theta_{X, \omega_{g_{t}^{\prime}}}$ (cf. Section 2) since $X$ lies in the center of $\eta_{r}(M)$ TZhu1]. Hence

$$
\begin{aligned}
& \lim _{i \rightarrow \infty} \int_{M} f_{t_{i}} e^{-f_{t_{i}}} d V_{g_{t_{i}}} \\
& =-\lim _{i \rightarrow \infty} \int_{M} \theta_{X, \omega_{g_{t_{i}}}} e^{\theta_{X, \omega_{g_{t_{i}}}} \omega_{g_{t_{i}}}^{n}=-N_{X}\left(c_{1}(M)\right) .}
\end{aligned}
$$

By combining (3.12) and (3.14), we get

$$
\begin{aligned}
& \lim _{i \rightarrow \infty} \lambda\left(g_{t_{i}}\right)=\lim _{i \rightarrow \infty} \int_{M}\left[\frac{1}{2}\left(R\left(g_{t_{i}}\right)+\left|\nabla f_{t_{i}}\right|^{2}\right)+f_{t_{i}}\right] e^{-f_{t_{i}}} d V_{g_{t_{i}}} \\
& =n V-N_{X}\left(c_{1}(M)\right) .
\end{aligned}
$$

Therefore, by using the monotonicity of $\lambda\left(g_{t}\right)$ along the flow $\left(g_{t} ; g\right)$, we obtain (3.1). 
It was showed in [TZhu4] that a Kähler-Ricci soliton is a local maximizer of $\lambda(\cdot)$ in the Kähler class $2 \pi c_{1}(M)$. Using this together with Corollary 1.5, one may guess that a Kähler-Ricci soliton is a global maximizer of $\lambda(\cdot)$. More generally, according to Corollary 3.2 , we propose the following conjecture.

Conjecture 3.4. Suppose that the modified Mabuchi's K-energy is bounded from below. Then

$$
\sup _{\omega_{g^{\prime}} \in 2 \pi c_{1}(M)} \lambda\left(g^{\prime}\right)=(2 \pi)^{-n}\left[n V-N_{X}\left(c_{1}(M)\right)\right]
$$

\section{Improvement of Lemma 3.3}

In this section, we use Perelman's backward heat flow to improve the estimates (b) and (c) in Lemma 3.3 independent of $t$. Moreover, we show that the gradient estimate of $f_{t}+h_{t}$ also holds. Although Lemma 3.3 is sufficient to be applied to prove Proposition 3.1 and Theorem 0.1, results of this section are independent of interests. We hope that these results will have applications in the future.

Fix any $t_{0} \geq 1$. We consider a backward heat equation in $t \in\left[t_{0}-1, t_{0}\right]$,

$$
\frac{\partial}{\partial t} f_{t_{0}}(t)=-\triangle f_{t_{0}}(t)+\left|\nabla f_{t_{0}}(t)\right|^{2}-\triangle h_{t}
$$

with an initial $f_{t_{0}}\left(t_{0}\right)=f_{t_{0}}$. Clearly, the equation preserves the normalizing condition $\frac{1}{V} \int_{M} e^{-f_{t_{0}}(t)} \omega_{g_{t}}^{n}=1$. Moreover, since $\triangle h_{t}$ and $f_{t}$ are uniformly bounded, by the maximum principle, we have

$$
\left\|f_{t_{0}}(t)\right\|_{C^{0}} \leq C(g), \quad \forall t \in\left[t_{0}-1, t_{0}\right] .
$$

Here the constant $C(g)$ depends only on the initial metric $g$ of (2.1).

Similarly to (1.5), we can compute

$$
\begin{aligned}
& \frac{d}{d t} W\left(g_{t}, f_{t_{0}}\right) \\
& =(2 \pi)^{-n} \int_{M}\left(\left|\partial \bar{\partial}\left(h_{t}+f_{t_{0}}(t)\right)\right|^{2}+\left|\partial \partial f_{t_{0}}(t)\right|^{2}\right) e^{-f_{t_{0}}(t)} \omega_{g_{t}}^{n} .
\end{aligned}
$$

By using (4.3), we want to prove

\section{Lemma 4.1.}

$$
\left\|f_{t}+h_{t}-c_{t}\right\|_{L^{2}\left(M, g_{t}\right)} \rightarrow 0, \text { as } t \rightarrow \infty,
$$

where $c_{t}=\frac{1}{V} \int_{M}\left(f_{t}+h_{t}\right) e^{h_{t}} \omega_{g_{t}}^{n}$.

Proof. First, by (4.3), one sees

$$
\begin{aligned}
\lambda\left(g_{t_{0}}\right)-\lambda\left(g_{t_{0}-1}\right) & \geq W\left(g_{t_{0}}, f_{t_{0}}\left(t_{0}\right)\right)-W\left(g_{t_{0}-1}, f_{t_{0}}\left(t_{0}-1\right)\right) \\
& \geq(2 \pi)^{-n} \frac{1}{2 n} \int_{t_{0}-1}^{t_{0}} \int_{M}\left|\triangle\left(f_{t_{0}}(t)+h_{t}\right)\right|^{2} e^{-f_{t_{0}}(t)} \omega_{g_{t}}^{n} d t .
\end{aligned}
$$

It follows that

$$
\int_{t_{0}-1}^{t_{0}} \int_{M}\left|\triangle\left(f_{t_{0}}(t)+h_{t}\right)\right|^{2} \omega_{g_{t}}^{n} d t \rightarrow 0, \text { as } t_{0} \rightarrow \infty
$$


Thus by using the weighted Poincaré inequality as in (3.4) in the last section, we will get

$$
\begin{aligned}
& \int_{t_{0}-1}^{t_{0}} d t \int_{M}\left(f_{t_{0}}(t)+h_{t}-c_{t_{0}}(t)\right)^{2} \omega_{g_{t}}^{n} \\
\leq & C\left(g_{0}\right)\left[\int_{t_{0}-1}^{t_{0}} d t \int_{M}\left|\triangle\left(f_{t_{0}}(t)+h_{t}\right)\right|^{2} \omega_{g_{t}}^{n}\right]^{1 / 2} \rightarrow 0, \text { as } t_{0} \rightarrow \infty,
\end{aligned}
$$

where $c_{t_{0}}(t)=\frac{1}{V} \int_{M}\left(f_{t_{0}}(t)+h_{t}\right) e^{h_{t}} \omega_{g_{t}}^{n}$.

Next, since $\frac{d h_{t}}{d t}=\Delta h_{t}+h_{t}-a_{t}$, where $a_{t}=\frac{1}{V} \int_{M} h_{t} e^{h_{t}} \omega_{g_{t}}^{n}$, by a straightforward calculation, we see

$$
\begin{aligned}
& \frac{d}{d t} \int_{M}\left(h_{t}+f_{t_{0}}(t)-c_{t_{0}}(t)\right)^{2} \omega_{g_{t}}^{n} \\
= & \int_{M}\left[2\left(h_{t}+f_{t_{0}}(t)-c_{t_{0}}(t)\right)\left(\triangle f_{t_{0}}-\left|\nabla f_{t_{0}}(t)\right|^{2}+h_{t}-a_{t}-\frac{d c_{t_{0}}}{d t}\right)\right. \\
& \left.-\left(h_{t}+f_{t_{0}}(t)-c_{t_{0}}(t)\right)^{2} \triangle h_{t}\right] \omega_{g_{t}}^{n} .
\end{aligned}
$$

Then, by Lemma 2.1, we get

$$
\begin{aligned}
& \left|\frac{d}{d t} \int_{M}\left(h_{t}+f_{t_{0}}(t)-c_{t_{0}}\right)^{2} \omega_{g_{t}}^{n}\right| \\
\leq & C+C \int_{M}\left(\left|\triangle\left(f_{t_{0}}(t)+h_{t}\right)\right|+\left|\nabla f_{t_{0}}(t)\right|^{2}+\left|\frac{d c_{t_{0}}(t)}{d t}\right|\right) \omega_{g_{t}}^{n} \\
\leq & C+C \int_{M}\left(\left|\nabla \bar{\nabla}\left(f_{t_{0}}(t)+h_{t}\right)\right|^{2}+\left|\frac{d c_{t_{0}}(t)}{d t}\right|\right) \omega_{g_{t}}^{n} .
\end{aligned}
$$

In this section, we will always denote $C$ to be a uniform constant which may be different in different places. Notice that

$$
\frac{d c_{t_{0}}}{d t}=\frac{1}{V} \int_{M}\left[\triangle f_{t_{0}}(t)-\left|\nabla f_{t_{0}}(t)\right|^{2}-\left(h_{t}+f_{t_{0}}(t)\right)\left(h_{t}+a_{t}\right)\right] e^{h_{t}} \omega_{g_{t}}^{n} .
$$

We can also estimate

$$
\left|\frac{d c_{t_{0}}}{d t}\right| \leq C+C \int_{M}\left|\nabla \bar{\nabla}\left(f_{t_{0}}(t)+h_{t}\right)\right|^{2} \omega_{g_{t}}^{n} .
$$

Hence we derive

$$
\begin{aligned}
& \left|\frac{d}{d t} \int_{M}\left(f_{t_{0}}(t)+h_{t}-c_{t_{0}}(t)\right)^{2} d v\right| \\
& \leq C+C \int_{M}\left|\nabla \bar{\nabla}\left(f_{t_{0}}(t)+h_{t}\right)\right|^{2} \omega_{g_{t}}^{n} .
\end{aligned}
$$

Therefore, according to

$$
\begin{aligned}
& \int_{t_{0}-1}^{t_{0}} d t \int_{M}\left|\nabla \bar{\nabla}\left(f_{t_{0}}(t)+h_{t}\right)\right|^{2} e^{-f_{t_{0}}(t)} \omega_{g_{t}}^{n} \\
\leq & (2 \pi)^{n}\left(\lambda\left(g_{t_{0}}\right)-\lambda\left(g_{t_{0}-1}\right)\right) \rightarrow 0, \text { as } t_{0} \rightarrow \infty,
\end{aligned}
$$

(4.5) and (4.6) will imply

$$
\left\|f_{t_{0}}(t)+h_{t}-c_{t_{0}}(t)\right\|_{L^{2}\left(g_{t}, M\right)} \rightarrow 0, \text { as } t_{0} \rightarrow \infty, \forall t \in\left[t_{0}-1, t_{0}\right] .
$$

Consequently, we get (4.4). 


\section{Proposition 4.2.}

$$
\left\|f_{t}+h_{t}\right\|_{C^{0}}+\left\|\nabla\left(f_{t}+h_{t}\right)\right\|_{g_{t}} \rightarrow 0, \text { as } t \rightarrow \infty .
$$

Proof. With the help of Lemma 4.1, by using the same argument in the proof of (c) in Lemma 3.3, we can prove that

$$
\left\|f_{t}+h_{t}\right\|_{C^{0}} \rightarrow 0, \text { as } t \rightarrow \infty
$$

So we suffice to prove

$$
\left\|\nabla\left(f_{t}+h_{t}\right)\right\|_{g_{t}} \rightarrow 0, \text { as } t \rightarrow \infty .
$$

We will use Moser's iteration to obtain (4.9) as in Lemma 7.2 in Appendix 1. We note by (4.8) and Theorem 7.1 in Appendix 1 that

$$
\int_{M}\left|\nabla q_{t}\right|^{2} \omega_{g_{t}}^{n}=\left|\int_{M}-\Delta\left(f_{t}+h_{t}\right)\left(f_{t}+h_{t}\right) \omega_{g_{t}}^{n}\right| \leq C\left\|f_{t}+h_{t}\right\|_{C^{0}} \rightarrow 0,
$$

where $q_{t}=f_{t}+h_{t}$ satisfies the equation

$$
\triangle q_{t}=\frac{1}{2}\left(\left|\nabla f_{t}\right|^{2}-2 f_{t}+\triangle h_{t}\right)+(2 \pi)^{n} V^{-1} \lambda\left(g_{t}\right)-n .
$$

Let $w_{t}=\left|\nabla q_{t}\right|^{2}$. Then, by the Bochner formula, we have

$$
\triangle w_{t}=\left|\nabla \nabla q_{t}\right|^{2}+\left|\nabla \bar{\nabla} q_{t}\right|^{2}+\nabla_{i} \triangle q_{t} \nabla_{\bar{i}} q_{t}+\nabla_{\bar{i}} \triangle q_{t} \nabla_{i} q_{t}+R_{i \bar{j}} \nabla_{\bar{i}} q_{t} \nabla_{j} q_{t} .
$$

Hence for any $p \geq 2$, it follows that

$$
\begin{aligned}
& \frac{4(p-1)}{p^{2}} \int_{M}\left|\nabla w_{t}^{p / 2}\right|^{2} \omega_{g_{t}}^{n}=-\int_{M} w_{t}^{p-1} \triangle q_{t} \omega_{g_{t}}^{n} \\
& =-\int_{M} w_{t}^{p-1}\left(\left|\nabla \nabla q_{t}\right|^{2}+\left|\nabla \bar{\nabla} q_{t}\right|^{2}\right) \omega_{g_{t}}^{n} \\
& -2 \operatorname{Re} \int_{M} q_{t}^{p-1} \nabla_{i} \triangle q_{t} \nabla_{\bar{i}} q_{t} \omega_{g_{t}}^{n}-\int_{M} q_{t}^{p-1} R_{i \bar{j}} \nabla_{\bar{i}} q_{t} \nabla_{j} q_{t} \omega_{g_{t}}^{n} .
\end{aligned}
$$

On the other hand, by Lemma 2.1 and Theorem 7.1, we estimate

$$
\begin{aligned}
& -2 \operatorname{Re} \int_{M} w_{t}^{p-1} \nabla_{i} \triangle q_{t} \nabla_{\bar{i}} w_{t} \omega_{g_{t}}^{n} \\
& =-\operatorname{Re} \int_{M} w_{t}^{q-1} \nabla_{i}\left(\left|\nabla f_{t}\right|^{2}-2 f+\Delta h_{t}\right) \nabla_{\bar{i}} q_{t} \omega_{g_{t}}^{n} \\
& =-\operatorname{Re} \int_{M}\left(\left|\nabla f_{t}\right|^{2}-2 f_{t}+\triangle h_{t}\right)\left(\frac{2(p-1)}{p} w_{t}^{\frac{p}{2}-1} \nabla_{i} w_{t}^{p / 2} \nabla_{\bar{i}} q_{t}+w_{t}^{q-1} \triangle q_{t}\right) \omega_{g_{t}}^{n} \\
& \leq C(g)\left[\int_{M} \frac{2(p-1)}{p} w_{t}^{\frac{p-1}{2}}\left|\nabla w_{t}^{p / 2}\right| \omega_{g_{t}}^{n}+\int_{M} w_{t}^{p-1}\left|\triangle q_{t}\right|\right] \omega_{g_{t}}^{n} \\
& \leq \frac{p-1}{p^{2}} \int_{M}\left|\nabla w^{p / 2}\right|^{2} \omega_{g_{t}}^{n}+C(g)^{\prime} p \int_{M} w^{p-1} \omega_{g_{t}}^{n}
\end{aligned}
$$


and

$$
\begin{aligned}
& -\int_{M} w_{t}^{p-1} R_{i \bar{j}} \nabla_{\bar{i}} q_{t} \nabla_{j} q_{t} \omega_{g_{t}}^{n} \\
= & -\int_{M} w_{t}^{p} \omega_{g_{t}}^{n}-\int_{M} w_{t}^{p-1} \nabla_{i} \nabla_{\bar{j}} h_{t} \nabla_{\bar{i}} q_{t} \nabla_{j} q_{t} \omega_{g_{t}}^{n} \\
= & -\int_{M} w_{t}^{p} \omega_{g_{t}}^{n}+\int_{M} w_{t}^{p-1} \nabla_{\bar{j}} h_{t}\left(\nabla_{\bar{i}} q_{t} \nabla_{i} \nabla_{j} q_{t} \omega_{g_{t}}^{n}+\Delta q_{t} \nabla_{j} q_{t}\right) \omega_{g_{t}}^{n} \\
& +\frac{2(p-1)}{p} \int_{M} w_{t}^{\frac{p}{2}-1} \nabla_{i} w_{t}^{p / 2} \nabla_{\bar{j}} h_{t} \nabla_{\bar{i}} q_{t} \nabla_{j} q \omega_{g_{t}}^{n} \\
\leq & \int_{M} w_{t}^{p-1}\left(\left|\nabla \nabla q_{t}\right|^{2}+\frac{1}{2}\left|\nabla \bar{\nabla} q_{t}\right|^{2}\right) \omega_{g_{t}}^{n}+\frac{p-1}{p^{2}} \int_{M}\left|\nabla w_{t}^{p / 2}\right|^{2} \omega_{g_{t}}^{n} \\
& +C(g)(p-1) \int_{M} w_{t}^{p} \omega_{g_{t}}^{n} .
\end{aligned}
$$

Then substituting the above two inequalities into (4.11), we get

$$
\int_{M}\left|\nabla w_{t}^{p / 2}\right|^{2} \omega_{g_{t}}^{n} \leq C(g)(p-1)^{2} \int_{M} w_{t}^{p-1} \omega_{g_{t}}^{n}, \quad \forall p \geq 2 .
$$

By using Zhang's Sobolev inequality Zha, we deduce

$$
\left(\int_{M} w_{t}^{p \nu}\right)^{1 / \nu} \omega_{g_{t}}^{n} \leq C(g) C_{s}(q-1)^{2} \int_{M} w_{t}^{p-1} \omega_{g_{t}}^{n}, \quad \forall p \geq 2,
$$

where $\nu=\frac{n}{n-1}$. To run the iteration we put $p_{0}=1$ and $p_{k+1}=p_{k} \nu+\nu, k \geq 0$. Hence

$$
\begin{aligned}
\left\|w_{t}\right\|_{L^{p_{k+1}}} & \leq\left(C C_{s}\right)^{\frac{1}{p_{k}+1}} p_{k}^{\frac{2}{p_{k}+1}}\|w\|_{L^{p_{k}}}^{\frac{p_{k}}{p_{k}+1}} \\
& \leq\left(C C_{s}\right)^{\sum_{i=0}^{i=k} \frac{\nu^{k-i}}{p_{k}+1}} \prod_{i=0}^{i=k} p_{i}^{\frac{2 \nu}{p_{k}+1}}\left\|w_{t}\right\|_{L^{1}}^{\prod \frac{p_{i}}{p_{i}+1}} \\
& \leq C(n, g) C_{s}^{\frac{n}{2}}\left\|w_{t}\right\|_{L^{1}}^{\gamma(n)}
\end{aligned}
$$

for a constant $\gamma(n)$ depending only on $n$, where we have used the fact that $p_{k} \leq 2 \nu^{k}$ for $k \geq 1$. Therefore by (4.10) we prove

$$
\left\|w_{t}\right\|_{C^{0}} \leq C(n, g) C_{s}^{\frac{n}{2}}\left\|w_{t}\right\|_{L^{1}}^{\gamma(n)} \rightarrow 0, \text { as } t \rightarrow \infty .
$$

\section{Another Version of $N_{X}\left(\omega_{g}\right)$}

Let $Y \in \eta_{r}(M)$ so that $\operatorname{Im}(Y)$ generates a one-parameter compact subgroup of

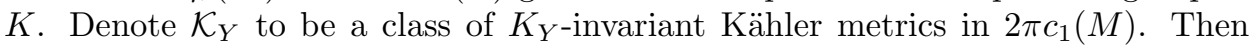
according to the proof of Proposition 1.4, we actually prove

$$
\sup _{g \in \mathcal{K}_{Y}} \lambda(g) \leq(2 \pi)^{-n}\left[n V-\tilde{F}_{Y}(Y)-N_{Y}\left(c_{1}(M)\right)\right],
$$

where

and

$$
\tilde{F}_{Y}(Y)=\int_{M} Y\left(h_{g}-\theta_{Y, \omega}\right) e^{\theta_{Y, \omega_{g}} \omega_{g}^{n}}
$$

$$
N_{Y}\left(c_{1}(M)\right)=\int_{M} \theta_{Y, \omega_{g}} e^{\theta_{Y, \omega_{g}} \omega_{g}^{n}}
$$

are both holomorphic invariants of $M$. In this section, we want to show 
Proposition 5.1. Let $H(Y)=\tilde{F}_{Y}(Y)+N_{Y}\left(c_{1}(M)\right)$. Then

$$
\sup _{Y \in \eta_{r}(M)} H(Y)=N_{X}\left(c_{1}(M)\right),
$$

where $X$ is the extremal vector field determined in Section 1.

Proof. Choose a constant $c_{Y}$ so that $\hat{\theta}_{Y, \omega_{g}}=\theta_{Y, \omega_{g}}+c_{Y}$ satisfies a normalization condition

$$
\int_{M} \hat{\theta}_{Y, \omega_{g}} e^{h_{g}} \omega_{g}^{n}=0
$$

Then $\hat{\theta}_{Y, \omega_{g}}$ satisfies the equation

$$
\Delta \hat{\theta}_{X, \omega_{g}}+X\left(h_{g}\right)+\hat{\theta}_{X, \omega_{g}}=0 .
$$

Thus using integration by parts, we have

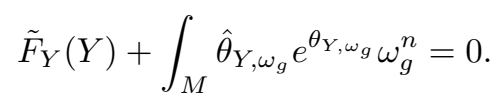

It follows that

$$
H(Y)=-c_{Y} V=\int_{M} \theta_{Y, \omega_{g}} e^{h_{g}} \omega_{g}^{n} .
$$

We compute the first variation of $H(Y)$ in $\eta_{r}(M)$. By the definition of $\theta_{Y+t Y^{\prime}}$, we see that there exist constants $b(t)$ such that $\theta_{Y+t Y^{\prime}}=\theta_{Y}+t \theta_{Y^{\prime}}+b(t)$. Since

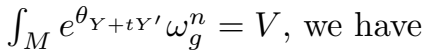

$$
e^{-b(t)}=\frac{1}{V} \int_{M} e^{\theta_{Y}+t \theta_{Y^{\prime}}} \omega_{g}^{n}
$$

Thus we get

$$
\left.\frac{d H\left(Y+t Y^{\prime}\right)}{d t}\right|_{t=0}=\int_{M} \theta_{Y^{\prime}} e^{h_{g}} \omega_{g}^{n}-\int_{M} \theta_{Y^{\prime}} e^{\theta_{Y}} \omega_{g}^{n}=\tilde{F}_{Y}\left(Y^{\prime}\right) .
$$

Therefore, by [TZhu2], we see that there exists a unique critical $X \in \eta_{r}(M)$ of $H(\cdot)$ such that

$$
\tilde{F}_{X}\left(Y^{\prime}\right)=F_{X}\left(Y^{\prime}\right) \equiv 0, \forall Y^{\prime} \in \eta_{r}(M) .
$$

Similarly, we have

$$
\theta_{t Y+(1-t) Y^{\prime}}=t \theta_{Y}+(1-t) \theta_{Y^{\prime}}+b(t)^{\prime}, \forall t \in[0,1]
$$

for some constants $b(t)^{\prime}$. Then

$$
\begin{aligned}
V=\int_{M} e^{\theta_{t Y+(1-t) Y^{\prime}} \omega_{g}^{n}} & =e^{b(t)^{\prime}} \int_{M} e^{t \theta_{Y}+(1-t) \theta_{Y^{\prime}}} \omega_{g}^{n} \\
& \leq e^{b(t)^{\prime}}\left[t \int_{M} e^{\theta_{Y}} \omega_{g}^{n}+(1-t) \int_{M} e^{\theta_{Y^{\prime}}} \omega_{g}^{n}\right] \\
& =e^{b(t)^{\prime}} V .
\end{aligned}
$$

Thus $b(t)^{\prime} \geq 0$. Consequently

$$
H(t X+(1-t) Y) \geq t H(X)+(1-t) H(Y) .
$$

This means that $H(\cdot)$ is a concave functional on $\eta_{r}(M)$. It follows that $X$ is a global maximizer of $H(\cdot)$. Therefore we prove the proposition by using the fact that $H(X)=N_{X}\left(c_{1}(M)\right)$. 
Corollary 5.2. Let $\mathcal{K}_{K}$ be a class of $K$-invariant Kähler metrics in $2 \pi c_{1}(M)$. Suppose that

$$
\sup _{g \in \mathcal{K}_{K}} \lambda(g)<\inf _{Y \in \eta_{r}(M)}(2 \pi)^{-n}\left[n V-F_{Y}(Y)-N_{Y}\left(c_{1}(M)\right)\right] .
$$

Then $(M, J)$ could not admit any Kähler-Ricci soliton. Furthermore, the modified Mabuchi's K-energy could not be bounded from below.

Proof. The first part of the corollary follows from Proposition 5.1 and Corollary 1.5. The second part follows from Proposition 5.1 and Corollary 3.2 .

The above corollary gives a new obstruction to the existence of Kähler-Ricci solitons.

\section{Proof of Theorem 0.1}

In this section, we will modify the proof of the Main Theorem in TZhu5 to prove Theorem 0.1. The proof in TZhu5] depends on a generalized uniqueness theorem for Kähler-Einsteins recently proved by Chen and Sun in CS. Here we avoid using Chen-Sun's theorem so that we can generalize the proof to the case of Kähler-Ricci solitons by applying Proposition 3.1.

As in [TZhu5], we write an initial Kähler form $\omega_{g}$ of Kähler-Ricci flow (2.2) by

$$
\omega_{g}=\omega_{\varphi}=\omega_{g_{K S}}+\sqrt{-1} \partial \bar{\partial} \varphi \in 2 \pi c_{1}(M)
$$

for a Kähler potential $\varphi$ on $M$. We define a path of Kähler forms

$$
\omega_{g^{s}}=\omega_{s \varphi}=\omega_{g_{K S}}+s \sqrt{-1} \partial \bar{\partial} \varphi
$$

and set

$$
\begin{aligned}
I=\{s \in[0,1] \mid & \left(g_{t}^{s} ; g^{s}\right) \text { converges to a Kähler-Ricci soliton } \\
& \text { exponentially in } \left.C^{\infty} \text { in the sense of Kähler potentials }\right\} .
\end{aligned}
$$

Clearly, $I$ is not empty by the assumption of the existence of Kähler-Ricci solitons on $M$. We want to show that $I$ is in fact both open and closed. Then it follows that $I=[0,1]$. This will finish the proof of Theorem 0.1

The openness of $I$ is related to the following stability theorem of Kähler-Ricci flow, which was proved in [Zhu2].

Lemma 6.1. Let $(M, J)$ be a compact Kähler manifold which admits a Kähler-Ricci soliton $\left(g_{K S}, X\right)$. Let $\psi$ be a Kähler potential of a $K_{X}$-invariant initial metric $g$ of (2.2). Then there exists a small $\epsilon$ such that if

$$
\|\psi\|_{C^{3}(M)} \leq \epsilon
$$

the solution $g(t, \cdot)$ of (2.2) converges to a Kähler-Ricci soliton with respect to $X$ in $C^{\infty}$ in the sense of Kähler potentials. Moreover, the convergence can be made fast exponentially.

For the convenience of the readers, we will give another proof to Lemma 6.1 in Appendix 2 by using a similar argument in the proof of closedness of $I$ later in this section. We also note that Lemma 6.1] is still true if the $K_{X}$-invariant condition is removed for the initial metric $g$ [hu2]. But we do not know whether or not the convergence is exponentially fast. 
Proof of openness of $I$. Suppose that $s_{0} \in I$. Then by the uniqueness of KählerRicci solitons [TZhu1, the flow $\left(g_{t}^{s_{0}} ; g^{s_{0}}\right)$ converges to $g_{K S}$ after a holomorphism transformation in $\operatorname{Aut}_{r}(M)$. Namely, there exists a $\sigma \in \operatorname{Aut}_{r}(M)$ such that $\sigma^{\star} \omega_{g_{t}^{s_{0}}}=\omega_{K S}+\sqrt{-1} \partial \bar{\partial}\left(\phi_{t}^{s_{0}}\right)_{\sigma}$ with property

$$
\left\|\left(\phi_{t}^{s_{0}}\right)_{\sigma}\right\|_{C^{k}(M)} \leq C_{k} e^{-\alpha_{k} t},
$$

where $C_{k}, \alpha_{k}>0$ are two uniform constants. Then we can choose $T$ sufficiently large such that

$$
\left\|\left(\phi_{T}^{s_{0}}\right)_{\sigma}\right\|_{C^{3}(M)}<\frac{\delta}{2}
$$

where $\delta$ is the small number determined in Lemma 6.1 Since the Kähler-Ricci flow is stable in any fixed finite time, there is a small $\epsilon>0$ such that

$$
\left\|\hat{\phi}_{T}^{s}-\left(\phi_{T}^{s_{0}}\right)_{\sigma}\right\|_{C^{3}(M)}<\frac{\delta}{2}, \forall s \in\left[s_{0}, s_{0}+\epsilon\right],
$$

where $\hat{\phi}_{T}^{s}$ is a Kähler potential of the evolved Kähler metric $\hat{g}_{T}^{s}$ of the Kähler-Ricci flow $\left(\hat{g}_{t}^{s} ; \sigma^{\star} g^{s}\right)$ at time $T$. Hence, we have

$$
\left\|\hat{\phi}_{T}^{s}\right\|_{C^{3}(M)}<\delta, \forall s \in\left[s_{0}, s_{0}+\epsilon\right] .
$$

Then the flow $\left(g_{t} ; \hat{g}_{T}^{s}\right)$ with initial $\hat{g}_{T}^{s}$ will converge to a Kähler-Ricci soliton exponentially in $C^{\infty}$ according to Lemma 6.1. Consequently, the flow $\left(g_{t}^{s} ; g^{s}\right)$ converges to a Kähler-Ricci soliton exponentially. This shows $s \in I$ for any $s \in\left[s_{0}, s_{0}+\epsilon\right]$.

Let $\phi_{t}^{s}$ be a family of Kähler potentials of the evolved Kähler metric $g_{t}^{s}$ of the Kähler-Ricci flow $\left(g_{t}^{s} ; g^{s}\right)$. To make the potentials $\phi_{t}^{s}$ smaller so that they can be controlled, we need the following lemma, which was proved in [TZhu1.

Lemma 6.2. Let $M$ be a compact Kähler manifold which admits a Kähler-Ricci soliton $\left(g_{K S}, X\right)$. Let $\varphi$ be a $K_{X}$-invariant Kähler potential and $\varphi_{\gamma}=\gamma^{\star} \varphi+\rho_{\gamma}$ for any $\gamma \in \operatorname{Aut}_{r}(M)$, where $\rho_{\gamma}$ is defined by $\gamma^{\star} \omega_{K S}=\omega_{K S}+\sqrt{-1} \partial \bar{\partial} \rho_{\gamma}$ with a normalization condition $\int_{M} e^{-\rho_{\gamma}} \omega_{K S}^{n}=\int_{M} \omega_{K S}^{n}$. Then there exists a unique $\sigma \in$ $\operatorname{Aut}_{r}(M)$ such that $\varphi_{\sigma} \in \Lambda^{\perp}\left(\omega_{K S}\right)$ with property

$$
J\left(\varphi_{\sigma}\right)=\inf _{\gamma \in \operatorname{Aut}_{r}(M)} J\left(\varphi_{\gamma}\right),
$$

where $\Lambda^{\perp}\left(\omega_{K S}\right)$ is an orthogonal space-to-kernel space of the linear operator $\left(\Delta_{g_{K S}}+\right.$ $X+I d)(\psi)$ and

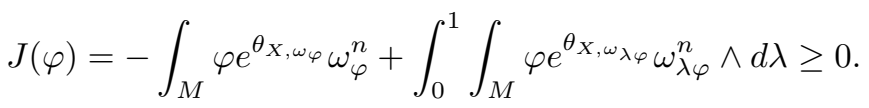

Moreover,

$$
\|\sigma-I d\| \leq C\left(\|\varphi\|_{C^{5}(M)}\right)
$$

where $\|\sigma-I d\|$ denotes the distance norm in Lie group $\operatorname{Aut}_{r}(M)$.

Proof of closedness of $I$. By the openness of $I$, we see that there exists a $\tau_{0} \leq 1$ with $\left[0, \tau_{0}\right) \subset I$. We need to show that $\tau_{0} \in I$. In fact we want to prove that for any $\delta>0$ there exists a large $T$ such that

$$
\left\|\left(\phi_{t}^{s}\right)_{\sigma_{s, t}}\right\|_{C^{5}(M)} \leq \delta, \forall t \geq T \text { and } s<\tau_{0},
$$

where $\sigma_{s, t}$ are some holomorphisms in $\operatorname{Aut}_{r}(M)$. We will use an argument by contradiction as in [TZhu5]. On the contrary, by Lemma 6.2 one can find a sequence of evolved Kähler metrics $g_{t_{i}}^{s_{i}}$ of Kähler-Ricci flows $\left(g_{t}^{s_{i}} ; g^{s_{i}}\right)$, where $s_{i} \rightarrow \tau_{0}$ and 
$t_{i} \rightarrow \infty$, and a sequence of unique holomorphisms $\sigma_{s_{i}, t_{i}} \in \operatorname{Aut}_{r}(M)$ for pairs $\left(s_{i}, t_{i}\right)$ such that $\left(\phi_{t_{i}}^{s_{i}}\right)_{\sigma_{s_{i}, t_{i}}} \in \Lambda^{\perp}\left(\omega_{K S}\right)$ and

$$
\left\|\left(\phi_{t_{i}}^{s_{i}}\right)_{\sigma_{s_{i}, t_{i}}}\right\|_{C^{5}(M)} \geq \delta_{0}
$$

for some constant $\delta_{0}>0$. On the other hand, since the Kähler-Ricci flow $\left(g_{t}^{s} ; g^{s}\right)$ $\left(s<\tau_{0}\right)$ converges to some Kähler-Ricci soliton, by the uniqueness of the KählerRicci solitons [TZhu1], there exists a unique $\sigma_{s} \in \operatorname{Aut}_{r}(M)$ for each $s$ such that

$$
\lim _{t \rightarrow \infty}\left\|\left(\phi_{t}^{s}\right)_{\sigma_{s}}\right\|_{C^{5}(M)}=0 .
$$

It follows by Lemma 6.2 that

$$
\lim _{t \rightarrow \infty}\left\|\left(\phi_{t}^{s}\right)_{\sigma_{s, t}}\right\|_{C^{5}(M)}=0,
$$

where $\left(\phi_{t}^{s}\right)_{\sigma_{s, t}} \in \Lambda^{\perp}\left(\omega_{K S}\right)$. Thus by (6.3), we may further assume that $\phi_{t_{i}}^{s_{i}}$ satisfies

$$
\left\|\left(\phi_{t_{i}}^{s_{i}}\right)_{\sigma_{s_{i}, t_{i}}}\right\|_{C^{5}(M)} \leq 2 \delta_{0} \text {. }
$$

We claim that there exists a subsequence $\left(\phi_{t_{i}}^{s_{i}}\right)_{\sigma_{s_{i}, t_{i}}}$ (still used by $\left(\phi_{t_{i}}^{s_{i}}\right)_{\sigma_{s_{i}, t_{i}}}$ ) of $\left(\phi_{t_{i}}^{s_{i}}\right)_{\sigma_{s_{i}, t_{i}}}$ converging to a potential $\phi_{\infty} \in \Lambda^{\perp}\left(\omega_{K S}\right)$ with property

$$
\delta_{0} \leq\left\|\phi_{\infty}\right\|_{C^{5}(M)} \leq 2 \delta_{0} .
$$

In fact, we will show that $\left(\phi_{t_{i}}^{s_{i}}\right)_{\sigma_{s_{i}, t_{i}}}$ is $C^{k}$-convergent to $\phi_{\infty}$ for any integer $k \geq 0$. Then the claim follows.

By (2.3), $\left(\phi_{t}^{s}\right)$ satisfies the equation

$$
\frac{\partial \phi^{\prime}}{\partial t}=\log \frac{\omega_{\phi^{\prime}}^{n}}{\omega_{K S}^{n}}+\phi^{\prime}+X\left(\phi^{\prime}\right), \phi^{\prime}(0, \cdot)=s \varphi+c,
$$

where $\omega_{\phi^{\prime}}=\omega_{K S}+\sqrt{-1} \partial \bar{\partial} \phi^{\prime}$. Then $\left(\phi_{t_{i}}^{s_{i}}\right)_{\sigma_{s_{i}, t_{i}}}$ is a solution of the following equation at time $t_{i}$ :

$$
\frac{\partial \phi}{\partial t}=\log \frac{\omega_{\phi}^{n}}{\omega_{K S}^{n}}+\phi+X(\phi), \omega_{\phi(0, \cdot)}=\left(\sigma_{s_{i}, t_{i}}\right)^{\star} \omega_{s_{i} \varphi} .
$$

Since the modified K-energy $\mu(\cdot)$ is bounded from below and $h_{\phi^{\prime}}$ is uniformly bounded by Lemma 2.1, as in TZhu3, (or TZhu5]), one can choose a suitable $c$ such that

It follows that

$$
\left|\frac{\partial \phi^{\prime}}{\partial t}\right|<C\left(\omega_{s \varphi}\right) \leq C
$$

In particular,

$$
\left|\frac{\partial \phi}{\partial t}\right| \leq\left|\frac{\partial \phi^{\prime}}{\partial t}\right|+2 \sup _{\omega_{\psi} \in \mathcal{K}_{X}} \theta_{X, \omega_{\psi}}<C^{\prime} .
$$

$$
\left|\frac{\partial \phi}{\partial t}\right|<C^{\prime}, \forall t \in\left(t_{i}-\frac{1}{2}, t_{i}+\frac{1}{2}\right) .
$$

(6.7) implies

$$
|\phi(t, \cdot)|<C^{\prime}+2 \delta_{0}, \forall t \in\left(t_{i}-\frac{1}{2}, t_{i}+\frac{1}{2}\right),
$$

because of

$$
\left|\phi\left(t_{i}, \cdot\right)\right|=\left|\left(\phi_{t_{i}}^{s_{i}}\right)_{\sigma_{s_{i}, t_{i}}}\right| \leq 2 \delta_{0} .
$$

By (6.7) and (6.8), we can apply Proposition 9.1 in Appendix 3 to see that

$$
\|\phi(t, \cdot)\|_{C^{k}(M)} \leq C_{k}, \forall t \in\left(t_{i}-\frac{1}{4}, t_{i}+\frac{1}{2}\right) .
$$


In particular, $\left(\phi_{t_{i}}^{s_{i}}\right)_{\sigma_{s_{i}, t_{i}}}$ is uniformly $C^{k}$-bounded, and consequently it is $C^{k}$-convergent to $\phi_{\infty}$ for any integer $k \geq 0$.

Next we want to show that

$$
\lambda\left(\omega_{\phi_{\infty}}\right)=\lambda\left(g_{K S}\right)=(2 \pi)^{-n}\left(n V-N_{X}\left(c_{1}(M)\right)\right) .
$$

First we note that the modified $K$-energy is bounded from below since $M$ admits a Kähler-Ricci soliton [TZhu2]. Then by Proposition 3.1] and the monotonicity of $\lambda\left(g_{t}^{\tau_{0}}\right)$, we see that for any $\epsilon>0$, there exists a large $T>0$ such that

$$
\lambda\left(g_{t}^{\tau_{0}}\right) \geq(2 \pi)^{-n}\left(n V-N_{X}\left(c_{1}(M)\right)\right)-\frac{\epsilon}{2}, \forall t \geq T .
$$

Since the Kähler-Ricci flow is stable in finite time and $\lambda\left(g_{t}^{s}\right)$ is monotonic in $t$, there is a small $\delta>0$ such that for any $s \geq \tau_{0}-\delta$, we have

$$
\lambda\left(g_{t}^{s}\right) \geq(2 \pi)^{-n}\left(n V-N_{X}\left(c_{1}(M)\right)\right)-\epsilon, \forall t \geq T .
$$

Since $s_{i} \rightarrow \tau_{0}$ and $t_{i} \rightarrow \infty$, we conclude that

$$
\lim _{s_{i} \rightarrow \tau_{0}, t_{i} \rightarrow \infty} \lambda\left(\sigma_{s_{i}, t_{i}}^{\star} g_{t_{i}}^{s_{i}}\right)=\lim _{s_{i} \rightarrow \tau_{0}, t_{i} \rightarrow \infty} \lambda\left(g_{t_{i}}^{s_{i}}\right)=(2 \pi)^{-n}\left(n V-N_{X}\left(c_{1}(M)\right)\right) .
$$

By the continuation of $\lambda(\cdot)$, we will get (6.9) .

Now by Corollary 1.5) together with (6.9), we see that $\omega_{\phi_{\infty}}$ is a global maximizer of $\lambda(\cdot)$ in $\mathcal{K}_{X}$. Then by the monotonicity of $\lambda(\cdot)$, it is easy to show that $\omega_{\phi_{\infty}}$ is a Kähler-Ricci soliton if we compute the first variation of $\lambda(\cdot)$ along the Kähler-Ricci flow (2.1) with the initial metric $\omega_{\phi_{\infty}}$ as done in (1.5). Thus by the uniqueness result for Kähler-Ricci solitons [TZhu1, TZhu2, we get

$$
\omega_{\phi_{\infty}}=\sigma^{\star} \omega_{K S}
$$

where $\sigma \in \operatorname{Aut}_{r}(M)$. Since $\phi_{\infty} \in \Lambda^{\perp}\left(\omega_{K S}\right)$, by Lemma 6.2, $\phi_{\infty}$ must be zero. This is a contradiction to (6.5). The contradiction implies that (6.2) is true.

By (6.2), we see that for any $\delta>0$ there exists a large $T_{0}$ and $\sigma_{0} \in \operatorname{Aut}_{r}(M)$ such that

$$
\left\|\left(\phi_{T_{0}}^{\tau_{0}}\right)_{\sigma_{0}}\right\|_{C^{5}(M)} \leq \delta
$$

Then by Lemma 6.1, the Kähler-Ricci flow $\left(g_{t} ; \omega_{\left(\phi_{T_{0}}^{\tau_{0}}\right)_{\sigma_{0}}}\right)$ converges to a Kähler-Ricci soliton exponentially. It follows that the Kähler-Ricci flow $\left(g_{t} ; \sigma_{0}^{\star} g^{\tau_{0}}\right)$ converges to a Kähler-Ricci soliton exponentially and so does $\left(g_{t}^{\tau_{0}} ; g^{\tau_{0}}\right)$. Thus, we prove that $\tau_{0} \in I$.

\section{Appendix 1}

In [TZhu5], it was proved that the minimizer $f_{t}$ of the $W\left(g_{t}, \cdot\right)$-functional associated to the evolved Kähler metric $g_{t}$ of the Kähler-Ricci flow (2.1) is uniformly bounded (see also [TZha]). In this appendix, we show that the gradient of $f_{t}$ and $\triangle f_{t}$ are also uniformly bounded. Namely, we prove

Theorem 7.1. There is a uniform constant $C$ such that

$$
\left\|f_{t}\right\|_{C^{0}}+\left\|\nabla f_{t}\right\|_{g_{t}}+\left\|\triangle f_{t}\right\|_{C^{0}} \leq C, \forall t>0 .
$$

We will derive $\left\|\nabla f_{t}\right\|$ in Theorem 7.1 by studying a general nonlinear elliptic equation as follows:

$$
\triangle w(x)=w(x) F(x, w(x))
$$


where the Laplace operator $\triangle$ is associated to a Kähler metric $g$ in $2 \pi c_{1}(M)$ and $F$ is a smooth function on $M \times \mathbb{R}^{+}$, which satisfies a structure condition:

$$
-A-B t^{\alpha} \leq F(\cdot, t) \leq H(t) .
$$

Here $0 \leq A, B \leq \infty, 0 \leq \alpha<\frac{2}{n}$ are constants, and $H$ is a proper function on $\mathbb{R}^{+}$ which satisfies a growth control at 0 :

$$
\limsup _{t \rightarrow 0}(t H(t))<\infty .
$$

Lemma 7.2. Let $w$ be a positive solution of (7.1). Then

$$
\|\nabla w\|_{C^{0}} \leq C(n) C_{s}^{\frac{n}{2}}\left(\|\nabla h\|_{C^{0}}+\|w F\|_{C^{0}}\right)^{n}\left(\int_{M}\left(1+|\nabla w|^{2}\right) d V_{g}\right)^{1 / 2}
$$

where $C_{s}$ is a Sobelev constant of $g$ and $h$ is a Ricci potential of $g$.

Proof. We will use Moser's iteration to the $L^{p}$-estimate of $|\nabla w|$. By the Bochner formula, we have

$$
\begin{aligned}
\triangle|\nabla w|^{2} & =|\nabla \nabla w|^{2}+|\nabla \bar{\nabla} w|^{2}+\nabla_{i} \triangle w \nabla_{\bar{i}} w+\nabla_{i} w \nabla_{\bar{i}} \triangle w+R_{i \bar{j}} \nabla_{\bar{i}} w \nabla_{j} w \\
& =|\nabla \nabla w|^{2}+|\nabla \bar{\nabla} w|^{2}+\nabla_{i}(w F) \nabla_{\bar{i}} w+\nabla_{i} w \nabla_{\bar{i}}(w F)+R_{i \bar{j}} \nabla_{\bar{i}} w \nabla_{j} w .
\end{aligned}
$$

Put $\eta=|\nabla w|^{2}+1$. Then for $p \geq 2$, it follows that

$$
\begin{aligned}
& \frac{4(p-1)}{p^{2}} \int_{M}\left|\nabla \eta^{p / 2}\right|^{2} d V_{g} \\
= & -\int_{M} \eta^{p-1} \triangle \eta d V_{g} \\
= & -\int_{M} \eta^{p-1}\left(|\nabla \nabla w|^{2}+|\nabla \bar{\nabla} w|^{2}\right) d V_{g}-\int_{M} \eta^{p-1} R_{i \bar{j}} \nabla_{\bar{i}} w \nabla_{j} w d V_{g} \\
- & \int_{M} \eta^{p-1}\left(\nabla_{i}(w F) \nabla_{\bar{i}} w+\nabla_{\bar{i}}(w F) \nabla_{i} w\right) d V_{g} .
\end{aligned}
$$

The last term on the right hand side can be estimated as follows:

$$
\begin{aligned}
& -\int_{M} \eta^{p-1}\left(\nabla_{i}(w F) \nabla_{\bar{i}} w+\nabla_{\bar{i}}(w F) \nabla_{i} w\right) d V_{g} \\
= & \int_{M} w F\left(\nabla_{i} \eta^{p-1} \nabla_{\bar{i}} w+\nabla_{\bar{i}} \eta^{p-1} \nabla_{i} w+2 \eta^{p-1} \triangle w\right) d V_{g} \\
= & \frac{2(p-1)}{p} \int_{M} w F \eta^{\frac{p}{2}-1}\left(\nabla_{i} \eta^{p / 2} \nabla_{\bar{i}} w+\nabla_{\bar{i}} \eta^{p / 2} \nabla_{i} w\right) d V_{g} \\
& +2 \int_{M} w F \eta^{p-1} \triangle w d V_{g} \\
\leq & \frac{2(p-1)}{p^{2}} \int_{M}\left|\nabla \eta^{p / 2}\right|^{2} d V_{g}+2(p-1) p \int_{M}(w F)^{2} \eta^{p-2}(\eta-1) d V_{g} \\
& +\int_{M} \eta^{p-1}\left(\frac{(\triangle w)^{2}}{2 n}+2 n(w F)^{2}\right) d V_{g} \\
\leq & \frac{2(p-1)}{p^{2}} \int_{M}\left|\nabla \eta^{p / 2}\right|^{2} d V_{g}+\frac{1}{2 n} \int_{M} \eta^{p-1}(\triangle w)^{2} d V_{g} \\
& +2[p(p-1)+n]\|w F\|_{C^{0}}^{2} \int_{M} \eta^{p} d V_{g} .
\end{aligned}
$$


For the second term on the right hand side, we note that

$$
R_{i \bar{j}}=g_{i \bar{j}}+h_{i \bar{j}} \text {. }
$$

Then

$$
\begin{aligned}
& -\int_{M} \eta^{p-1} R_{i \bar{j}} \nabla_{\bar{i}} w \nabla_{j} w d V_{g} \\
= & \int_{M} \eta^{p-1} \nabla_{i} \nabla_{\bar{j}} h \nabla_{\bar{i}} w \nabla_{j} w d V_{g}-\int_{M} \eta^{p} d V_{g} \\
= & \frac{2(p-1)}{p} \int_{M} \eta^{p / 2-1} \nabla_{\bar{j}} h \nabla_{i} \eta^{p / 2} \nabla_{\bar{i}} w \nabla_{j} w d V_{g} \\
& +\int_{M} \eta^{p-1} \nabla_{\bar{j}} h\left(\triangle w \nabla_{j} w+\nabla_{\bar{i}} w \nabla_{i} \nabla_{j} w\right) d V_{g}-\int_{M} \eta^{p} d V_{g} .
\end{aligned}
$$

Thus

$$
\begin{aligned}
& -\int_{M} \eta^{p-1} R_{i j} \nabla_{i} w \nabla_{j} w d V_{g} \\
\leq & \frac{p-1}{p^{2}} \int_{M}\left|\nabla \eta^{p / 2}\right|^{2} d V_{g}+p(p-1)\|\nabla h\|_{C^{0}}^{2} \int_{M} \eta^{p-2}(\eta-1)^{2} d V_{g} \\
& +\frac{1}{2 n} \int_{M} \eta^{p-1}(\triangle w)^{2} d V_{g}+\frac{n}{2}\|\nabla h\|_{C^{0}}^{2} \int_{M} \eta^{p-1}(\eta-1) d V_{g} \\
& +\frac{1}{2 n} \int_{M} \eta^{p-1}|\nabla \nabla w|^{2} d V_{g}+\frac{n}{2}\|\nabla h\|_{C^{0}}^{2} \int_{M} \eta^{p-1}(\eta-1) d V_{g} \\
\leq & \frac{p-1}{p^{2}} \int_{M}\left|\nabla \eta^{p / 2}\right|^{2} d V_{g}+\frac{1}{2 n} \int_{M} \eta^{p-1}(\triangle w)^{2} d V_{g} \\
& +\frac{1}{2 n} \int^{p-1}|\nabla \nabla w|^{2} d V_{g}+[p(p-1)+n]\|\nabla h\|_{C^{0}}^{2} \int_{M} \eta^{p} d V_{g} .
\end{aligned}
$$

Substituting (7.6) and (7.7) into (7.5), we get

$$
\begin{aligned}
& \frac{p-1}{p^{2}} \int_{M}\left|\nabla \eta^{p / 2}\right|^{2} d V_{g} \\
\leq & -\int_{M} \eta^{p-1}\left(|\nabla \nabla w|^{2}+|\nabla \bar{\nabla} w|^{2}\right) d V_{g} \\
& +\frac{1}{n} \int_{M} \eta^{p-1}(\triangle w)^{2} d V_{g}+\frac{1}{2 n} \int_{M} \eta^{p-1}|\nabla \nabla w|^{2} d V_{g} \\
& +[2(p-1) p+n]\left(\|\nabla h\|_{C^{0}}^{2}+\|w F\|_{C^{0}}^{2}\right) \int_{M} \eta^{p} d V_{g} \\
\leq & C(n) p^{2}\left(\|\nabla h\|_{C^{0}}^{2}+\|w F\|_{C^{0}}^{2}\right) \int_{M} \eta^{p} d V_{g} .
\end{aligned}
$$

It follows that

$$
\int_{M}\left|\nabla \eta^{p / 2}\right|^{2} d V_{g} \leq C(n) p^{3}\left(\|\nabla h\|_{C^{0}}^{2}+\|w F\|_{C^{0}}^{2}\right) \int_{M} \eta^{p} d V_{g}, \quad \forall p \geq 2 .
$$

Therefore, by iteration, we derive that

$$
\sup \eta \leq C(n) D^{n / 2}\left(\int_{M} \eta^{2} d V_{g}\right)^{1 / 2}
$$

where $D=C_{s}\left(\|\nabla h\|_{C_{0}}^{2}+\|w F\|_{C^{0}}^{2}\right)$. This implies (17.4). 


\section{Proposition 7.3.}

$$
\|\nabla w\|_{C^{0}} \leq C\left(\|w\|_{L^{2}}\right)
$$

where the constant $C$ depends only on $n, C_{s}, A, B, \alpha, H, \operatorname{Vol}(g),\|\nabla h\|_{C^{0}}$ and $\|w\|_{L^{2}}$. Proof. First we note that by using the standard Moser's iteration to the equation

$$
\triangle w(x) \geq-A-B w^{\alpha},
$$

it is easy to see that

$$
\sup w \leq C\left(1+\|w\|_{L^{2}}^{\gamma}\right)
$$

for some constants $C$ and $\gamma$ which depend only on $n, C_{s}, A, B, \alpha, H$ and $\operatorname{Vol}(\mathrm{g})$. On the other hand, by (7.1), we have

$$
\int_{M}|\nabla w|^{2} d V_{g}=-\int_{M} w \triangle w d V_{g}=-\int_{M} w F d V_{g}
$$

Then we see that $\|\nabla w\|_{L^{2}}$ is bounded by $\|w\|_{L^{2}}$. Thus the proposition follows from Lemma 7.2

Since $v_{t}=e^{-\frac{f_{t}}{2}}$ satisfies (3.11) which is a type of the equation (7.1), then by Perelman's estimates (d) in Lemma 2.1 and Zhang's estimate for Sobelev constants associated to $g_{t}$ in [Zha] together with the $C^{0}$-estimate for $f_{t}$ in [TZhu5], we obtain a uniform gradient estimate for $v_{t}$ from Proposition 7.3 , and so for $f_{t}$. By equation (1.4), we also derive a a uniform Laplacian estimate for $f_{t}$. Thus Theorem 7.1 is true. Theorem 7.1 was used in Section 4.

\section{Appendix 2}

Proof of Lemma 6.1. As in Section 6, we need to prove that for any $\delta>0$ there exists an $\epsilon>0$ such that

$$
\left\|\left(\phi_{t}\right)_{\sigma_{t}}\right\|_{C^{5}(M)} \leq \delta, \forall t \geq 0
$$

as long as $\|\psi\|_{C^{3}(M)} \leq \epsilon$, where $\phi_{t}$ are potentials of evolved Kähler metrics in (2.2) with an initial Kähler metric $\omega_{\psi}$ and $\sigma_{t}$ are some holomorphisms in $\operatorname{Aut}_{r}(M)$. On the contrary, by Lemma 6.2. one can find a sequence of functions $\psi_{i}$ with $\left\|\psi_{i}\right\|_{C^{3}(M)} \rightarrow 0$ as $i \rightarrow \infty$ and a sequence of evolved Kähler metrics $g_{t_{i}}^{i}$ of KählerRicci flows $\left(g_{t}^{i} ; \omega_{\psi_{i}}\right)$, and a sequence of unique holomorphisms $\sigma_{t_{i}} \in \operatorname{Aut}_{r}(M)$ such that

$$
\left(\phi_{t_{i}}^{i}\right)_{\sigma_{t_{i}}} \in \Lambda^{\perp}\left(\omega_{K S}\right) \text { and }\left\|\left(\phi_{t_{i}}^{i}\right)_{\sigma_{t_{i}}}\right\|_{C^{5}(M)} \geq \delta_{0}>0
$$

for some constant $\delta_{0}$. Furthermore, by the stability of the Kähler-Ricci flow in finite time and Lemma 6.2, we may assume that $\phi_{t_{i}}^{i}$ satisfies

$$
\left\|\left(\phi_{t_{i}}^{i}\right)_{\sigma_{t_{i}}}\right\|_{C^{5}(M)} \leq 2 \delta_{0} .
$$

Then there exists a subsequence $\left(\phi_{t_{i}}^{i}\right)_{\sigma_{t_{i}}}$ (still used by $\left(\phi_{t_{i}}^{i}\right)_{\sigma_{t_{i}}}$ ) of $\left(\phi_{t_{i}}^{i}\right)_{\sigma_{t_{i}}}$ converging to a potential $\phi_{\infty} \in \Lambda^{\perp}\left(\omega_{K S}\right)$ with property

$$
\delta_{0} \leq\left\|\phi_{\infty}\right\|_{C^{5}(M)} \leq 2 \delta_{0} .
$$

In fact, (8.4) can be obtained by using the same argument as in the proof of (6.5) in Section 6.

On the other hand, by the monotonicity of $\lambda\left(g_{t}^{i}\right)$ in $t$ for each $i$, we have

$$
\lambda\left(\omega_{\phi_{\infty}}\right)=\lambda\left(g_{K S}\right)=(2 \pi)^{-n}\left(n V-N_{X}\left(c_{1}(M)\right) .\right.
$$


Then, by Corollary 1.5, we see that $\omega_{\phi_{\infty}}$ is a global maximizer of $\lambda(\cdot)$ in $\mathcal{K}_{X}$ and $\omega_{\phi_{\infty}}$ is a Kähler-Ricci soliton with respect to $X$. Thus by the uniqueness of Kähler-Ricci solitons [TZhu2, it follows that

$$
\omega_{\phi_{\infty}}=\sigma^{\star} \omega_{K S}
$$

for $\sigma \in \operatorname{Aut}_{r}(M)$. Since $\phi_{\infty} \in \Lambda^{\perp}\left(\omega_{K S}\right)$, by Lemma 6.2 $\phi_{\infty}$ must be zero. This is a contradiction to (8.4). The contradiction implies that (8.1) is true.

By (8.1), one can apply an argument in [TZhu3] to show that there exist another family of $\hat{\sigma}_{t}$ in $\operatorname{Aut}_{r}(M)$ such that $\left(\phi_{t}\right)_{\hat{\sigma}_{t}}$ are uniformly bounded in $C^{k}$ for any integer $k \geq 0$. Then by the uniqueness of Kähler-Ricci solitons and the monotonicity of modified $K$-energy, it follows that $\omega_{\left(\phi_{t}\right)_{\hat{\sigma}_{t}}}\left(\hat{\sigma}_{t}\right.$ may be modified) converges to $\omega_{g_{K S}}$ in $C^{\infty}$ in the sense of Kähler potentials. Since $\omega_{\left(\phi_{t}\right)_{\hat{\sigma}_{t}}}$ satisfies a modified equation of (2.2), we can use an argument in [Zhu2] (or [PSSW]) to show that $\omega_{\left(\phi_{t}\right)_{\hat{\sigma}_{t}}}$ converges to $\omega_{g_{K S}}$ exponentially in $C^{\infty}$ in the sense of Kähler potentials. It follows that the flow (2.2) converges to a Kähler-Ricci soliton exponentially with respect to $X$ in $C^{\infty}$ in the sense of Kähler potentials.

\section{Appendix 3}

In this appendix, we give an a priori estimate about the local regularity of the Kähler-Ricci flow which was used in Section 6.

Proposition 9.1. Let $(M, J)$ be a Fano manifold which admits a Kähler-Ricci soliton $\left(g_{K S}, X\right)$ with its Kähler form $\omega_{K S}$ in $2 \pi c_{1}(M)$. Let $\phi=\phi(t, \cdot)=\phi_{t}$ be a $K_{X}$-invariant solution of the Kähler-Ricci flow,

$$
\frac{\partial \phi}{\partial t}=\log \frac{\omega_{\phi}^{n}}{\omega_{K S}^{n}}+\phi+X(\phi), t \in(0,1) .
$$

Suppose that

$$
|\phi| \leq A \text { and }|\dot{\phi}|=\left|\frac{\partial \phi}{\partial t}\right| \leq A .
$$

Then for any integer $k \geq 0$ there exists a uniform $C_{k}=C_{k}\left(\omega_{K S}, A, k\right)$ such that

$$
\left\|\phi_{t}\right\|_{C^{k}(M)}<C_{k}, \forall t \in\left[\frac{1}{4}, 1\right) .
$$

We first prove

Lemma 9.2. Under the condition (9.2), it holds that

$$
n+\Delta \phi \leq e^{\frac{B}{t}}, \forall t \in(0,1) .
$$

Proof. Let $\Delta^{\prime}=\Delta_{t}^{\prime}$ be the Laplacian operator associated to $\omega_{\phi_{t}}$. Set

$$
F=\frac{\partial \phi}{\partial t}-X(\phi)-\phi
$$

Note that $F$ is uniformly bounded by (9.2). Then for sufficiently large $c$, using (9.1) and following the arguments in Ya, we compute that

$$
\begin{aligned}
& \Delta^{\prime}\left(e^{-\frac{c}{t}(\phi+A+1)}(n+\Delta \phi)\right) \\
\geq & e^{-\frac{c}{t}(\phi+A+1)}\left[\Delta F-C_{1}-\frac{c}{t} n(n+\Delta \phi)\right. \\
& \left.+\frac{c}{2 t}(n+\Delta \phi) \sum_{i=1}^{n} \frac{1}{1+\phi_{i \bar{i}}}\right],
\end{aligned}
$$


where we choose a local coordinate system at a given point $p \in M$ so that $\omega_{K S}=$ $\sqrt{-1} \sum_{i=1}^{n} d z^{i} \wedge d \bar{z}^{i}$ and $\phi_{i \bar{j}}=\delta_{i j} \phi_{i \bar{i}}$ at $p$. Note that

$$
\begin{aligned}
\Delta F= & \Delta \dot{\phi}-(n+\Delta \phi)+n-\Delta(X(\phi)) \\
\geq & \frac{\partial}{\partial t}(n+\Delta \phi)-\left(|\nabla X|_{\omega_{K S}}+1\right)(n+\Delta \phi) \\
& -\frac{c}{t}(n+\Delta \phi) \sup _{M} X(\phi)-e^{\frac{c}{t}(\phi+A+1)}\left(X\left(e^{-\frac{c}{t}(\phi+A+1)}(n+\Delta \phi)\right)\right) \\
\geq & \frac{\partial}{\partial t}(n+\Delta \phi)-\frac{C_{2} c}{t}(n+\Delta \phi) \\
& -e^{\frac{c}{t}(\phi+A+1)}\left(X\left(e^{-\frac{c}{t}(\phi+A+1)}(n+\Delta \phi)\right)\right) .
\end{aligned}
$$

Since

$$
\begin{aligned}
\sum_{i=1}^{n} \frac{1}{1+\phi_{i \bar{i}}} & \geq \prod_{i=1}^{n}\left(1+\phi_{i \bar{i}}\right)^{-\frac{1}{n-1}}(n+\Delta \phi)^{\frac{1}{n-1}} \\
& =e^{-\frac{F}{n-1}}(n+\Delta \phi)^{\frac{1}{n-1}} \\
& \geq \delta(n+\Delta \phi)^{\frac{1}{n-1}}
\end{aligned}
$$

and

$$
\begin{aligned}
& \frac{\partial}{\partial t}\left(e^{-\frac{c}{t}(\phi+A+1)}(n+\Delta \phi)\right) \\
= & e^{\frac{-c}{t}(\phi+A+1)} \frac{\partial}{\partial t}(n+\Delta \phi)-c\left(\frac{1}{t} \dot{\phi}-\frac{1}{t^{2}}(\phi+A+1)\right) e^{-\frac{c}{t}(\phi+A+1)}(n+\Delta \phi),
\end{aligned}
$$

we get from (9.3) and (9.4) that

$$
\begin{aligned}
& \left(\Delta^{\prime}-\frac{\partial}{\partial t}\right)\left(e^{-\frac{c}{t}(\phi+A+1)}(n+\Delta \phi)\right)+X\left(e^{-\frac{c}{t}(\phi+A+1)}(n+\Delta \phi)\right) \\
\geq & e^{-\frac{c}{t}(\phi+A+1)}\left(\frac{c}{C_{3} t}(n+\Delta \phi)^{1+\frac{1}{n-1}}-\frac{C_{4} c}{t^{2}}(n+\Delta \phi)-C_{5}\right) .
\end{aligned}
$$

Since $H=e^{-\frac{c}{t}(\phi+A+1)}(n+\Delta \phi)=0$ at $t=0$, one can apply the maximum principle to $H$ in (9.5) to see that there exist $t_{0}>0$ and $x_{0} \in M$ such that $\max _{M \times[0,1]} H=H\left(x_{0}, t_{0}\right)$ and

It follows that

$$
n+\left.\Delta \phi\right|_{\left(t_{0}, x_{0}\right)} \leq \frac{C_{6}}{t_{0}^{n-1}} .
$$

$$
\begin{aligned}
& \left.e^{-\frac{c}{t}(\phi+A+1)}(n+\Delta \phi)\right) \\
\leq & \left.e^{-\frac{c}{t}(\phi+A+1)}(n+\Delta \phi)\right)\left.\right|_{\left(t_{0}, x_{0}\right)} \leq e^{-\frac{c}{t_{0}}\left(\phi\left(t_{0}, x_{0}\right)+A+1\right)} \frac{C_{6}}{t_{0}^{n-1}} \leq C_{7} .
\end{aligned}
$$

This proves the lemma.

We also have

Lemma 9.3. Under the condition (9.2), the following is true:

i) the metric $\omega_{K S}+\sqrt{-1} \partial \bar{\partial} \phi_{t}$ is uniformly equivalent to $\omega_{K S}$ for any $t \geq t_{0}>0$;

ii) we have a uniform estimate

$$
\|\phi\|_{C^{3}(M)} \leq e^{\frac{\gamma}{t}}, \forall t \in(0,1),
$$

where $\gamma=\gamma\left(\omega_{K S}, A\right)$. 
Proof. (i) follows easily from equation (9.1) and Lemma 9.2. For the third derivative estimate, we consider Calabi's function

$$
S=g^{\prime i \bar{r}} g^{\prime s \bar{j}} g^{\prime k \bar{t}} \phi_{i \bar{j} k} \phi_{\bar{r} s \bar{t}},
$$

where $\left(g^{\prime} i \bar{j}\right)$ is the inverse of the Hermitian matrix function associated to the metric $\omega_{\phi}$. Note that $S$ is equivalent to the quantity

$$
g^{i \bar{r}} g^{s \bar{j}} g^{k \bar{t}} \phi_{i \bar{j} k} \phi_{\bar{r} s \bar{t}}, \quad \forall t \geq t_{0}>0
$$

where $g^{i \bar{r}}$ denotes the inverse of the Hermitian matrix function associated to the metric $\omega_{K S}$.

As in the proof of (9.5), by i) in Lemma 9.3 , it is easy to get

$$
\begin{aligned}
& \left(\Delta^{\prime}-\frac{\partial}{\partial t}\right)\left(e^{-\frac{2 \alpha}{t}}(n+\Delta \phi)\right)+X\left[e^{-\frac{2 \alpha}{t}}(n+\Delta \phi)\right] \\
\geq & C_{1} e^{-\frac{\alpha}{t}}(n+\Delta \phi)-C_{2} .
\end{aligned}
$$

Moreover, following Calabi's computation as in [Ya, by i) in Lemma 9.3. we can estimate

$$
\begin{aligned}
& \left(\Delta^{\prime}-\frac{\partial}{\partial t}\right)\left(e^{-\frac{2 \beta}{t}} S\right)+X\left(e^{-\frac{2 \beta}{t}} S\right) \\
\geq & -C_{3} e^{-\frac{\beta}{t}} S-C_{4} .
\end{aligned}
$$

Here $\alpha$ and $\beta$ are both sufficiently large numbers. By choosing $\beta>\alpha$ and another sufficiently large $A$, it follows that

$$
\begin{aligned}
& \left(\Delta^{\prime}-\frac{\partial}{\partial t}\right)\left(e^{-\frac{2 \beta}{t}} S+A e^{-\frac{2 \alpha}{t}}(n+\Delta \phi)\right)+X\left(e^{-\frac{2 \beta}{t}} S+A e^{-\frac{2 \alpha}{t}}(n+\Delta \phi)\right) \\
\geq & C_{5} e^{-\frac{\alpha}{t}} S-C_{6} .
\end{aligned}
$$

Thus by applying the maximum principle to the function $e^{-\frac{2 \beta}{t}} S+A e^{-\frac{2 \alpha}{t}}(n+\Delta \phi)$ in (9.8), we will get

$$
S \leq C_{7} e^{\frac{2 \beta}{t}}
$$

This implies (9.7).

Proposition 9.1 follows from Lemma 9.3 and the regularity theory to the parabolic equation (9.1).

\section{ACKNOWLEDGEMENTS}

The authors would like to thank the referee for suggestions that improved the presentation of our paper, particularly, the valuable discussions on Section 6 .

\section{REFERENCES}

[Ca] Huai Dong Cao, Deformation of Kähler metrics to Kähler-Einstein metrics on compact Kähler manifolds, Invent. Math. 81 (1985), no. 2, 359-372, DOI 10.1007/BF01389058. MR:799272 (87d:58051)

[CS] X.X. Chen and C. Sun, Calabi flow, geodesic rays, and uniqueness of constant scalar curvature Kähler metrics, arXiv:1004.2012v1, 2010.

[CTZ] Huai-Dong Cao, Gang Tian, and Xiaohua Zhu, Kähler-Ricci solitons on compact complex manifolds with $C_{1}(M)>0$, Geom. Funct. Anal. 15 (2005), no. 3, 697-719, DOI 10.1007/s00039-005-0522-y. MR2221147 (2007h:32031)

[Fu] A. Futaki, An obstruction to the existence of Einstein Kähler metrics, Invent. Math. 73 (1983), no. 3, 437-443, DOI 10.1007/BF01388438. MR718940 (84j:53072) 
[Ma] Toshiki Mabuchi, Some symplectic geometry on compact Kähler manifolds. I, Osaka J. Math. 24 (1987), no. 2, 227-252. MR909015 (88m:53126)

[Pe] G. Perelman, The entropy formula for the Ricci flow and its geometric applications, arxiv:math.DG/0211159.

[PSSW] D.H. Phong, J. Song, J. Sturm and B. Weinkove, The modified Kähler-Ricci flow and solitons, arxiv:0809.0941v1.

[Ro] O. S. Rothaus, Logarithmic Sobolev inequalities and the spectrum of Schrödinger operators, J. Funct. Anal. 42 (1981), no. 1, 110-120, DOI 10.1016/0022-1236(81)90050-1. MR620582 (83f:58080b)

[ST] Natasa Sesum and Gang Tian, Bounding scalar curvature and diameter along the Kähler Ricci flow (after Perelman), J. Inst. Math. Jussieu 7 (2008), no. 3, 575-587, DOI 10.1017/S1474748008000133. MR2427424 (2009c:53092)

[TZha] G. Tian and Z.L. Zhang, Degeneration of Kähler-Ricci Solitons, Int. Math. Res. Notices, (2011) doi:10.1093/imrn/rnr036

[TZhu1] Gang Tian and Xiaohua Zhu, Uniqueness of Kähler-Ricci solitons, Acta Math. 184 (2000), no. 2, 271-305, DOI 10.1007/BF02392630. MR1768112 (2001h:32040)

[TZhu2] Gang Tian and Xiaohua Zhu, A new holomorphic invariant and uniqueness of KählerRicci solitons, Comment. Math. Helv. 77 (2002), no. 2, 297-325, DOI 10.1007/s00014002-8341-3. MR1915043 (2003i:32042)

[TZhu3] Gang Tian and Xiaohua Zhu, Convergence of Kähler-Ricci flow, J. Amer. Math. Soc. 20 (2007), no. 3, 675-699, DOI 10.1090/S0894-0347-06-00552-2. MR2291916 (2007k:53107)

[TZhu4] G. Tian and X.H. Zhu, Perelman's W-functional and stability of Kähler-Ricci flow, arxiv:0801.3504v1.

[TZhu5] G. Tian and X.H. Zhu, Convergence of Kähler-Ricci flow on Fano manifolds,, J. Reine Angew. Math. 678 (2013), 223-245. DOI 10.1515.

[Ya] Shing Tung Yau, On the Ricci curvature of a compact Kähler manifold and the complex Monge-Ampère equation. I, Comm. Pure Appl. Math. 31 (1978), no. 3, 339-411, DOI 10.1002/cpa.3160310304. MR480350 (81d:53045)

[Zha] Qi S. Zhang, A uniform Sobolev inequality under Ricci flow, Int. Math. Res. Not. IMRN 17 (2007), Art. ID rnm056, 17, DOI 10.1093/imrn/rnm056. MR2354801 (2008g:53083)

[Zhu1] Xiaohua Zhu, Kähler-Ricci soliton typed equations on compact complex manifolds with $C_{1}(M)>0$, J. Geom. Anal. 10 (2000), no. 4, 759-774, DOI 10.1007/BF02921996. MR.1817785(2002c:32042)

[Zhu2] X.H. Zhu, Stability of Kähler-Ricci flow on a Fano manifold, Math. Ann. 356 (2013), 1425-1454. DOI 10.1007. MR3072807

[ZZ] Bin Zhou and Xiaohua Zhu, Relative $K$-stability and modified $K$-energy on toric manifolds, Adv. Math. 219 (2008), no. 4, 1327-1362, DOI 10.1016/j.aim.2008.06.016. MR2450612(2010b:32038)

School of Mathematical Sciences and BICMR, Peking University, Beijing, 100871, People's Republic of China - And - Department of Mathematics, Princeton University, Princeton, New Jersey 02139

E-mail address: tian@math.mit.edu

Beijing International Center for Mathematical Research, Peking University, BeiJing, 100871, PEOPle's Republic of ChinA

Current address: School of Mathematics and Systems Science, Beijing University of Aeronautics \& Astronautics, Beijing, 100191, People's Republic of China

E-mail address: zhangshj.1982@yahoo.com.cn

Department of Mathematics, Beijing Capital Normal University, Beijing, People's Republic OF CHinA

E-mail address: zhleigo@aliyun.com

School of Mathematical Sciences and BICMR, Peking University, Beijing, 100871, People's Republic of China

E-mail address: xhzhu@math.pku.edu.cn 